\title{
Selective Synthesis of Substituted 3-Aryl-2-phenyl-1,3-thiazolidin-4-one Sulfoxides and Sulfones by $S$-Oxidation with Oxone
}

\author{
Kevin C. Cannon ${ }^{1}$, Miguel Costa ${ }^{1}$, Matthew Pepper ${ }^{1}$, John Toovy $^{1}$, Rachel S. Selinsky ${ }^{2}$, Anthony F. Lagalante ${ }^{2}$ \\ ${ }^{1}$ Department of Chemistry, Pennsylvania State University, Abington Campus, Abington, Pennsylvania USA \\ ${ }^{2}$ Department of Chemistry, Villanova University, Villanova, Pennsylvania, USA \\ Correspondence: Kevin C. Cannon, Department of Chemistry, 1600 Woodland Road, Pennsylvania State University, \\ Abington Campus, Abington, Pennsylvania 19001, USA. Tel: 215-881-7468. E-mail: kcc10@psu.edu
}

Received: June 25, 2017 Accepted: July 11, 2017 Online Published: July 12, 2017

doi:10.5539/ijc.v9n4p1

URL: https://doi.org/10.5539/ijc.v9n4p1

\begin{abstract}
$S$-oxidation of 3-aryl-2-phenyl-1,3-thiazolidin-4-ones with Oxone ${ }^{\circledR}$ was investigated. For all compounds evaluated, selective oxidation to the sulfoxide was realized using 3 equivalents of Oxone ${ }^{\circledR}$ at room temperature. Alternatively, the sulfone was prepared selectively in most of the compounds evaluated at high temperature by increasing the equivalents of Oxone ${ }^{\circledR}$ used; the extent of this selectivity was affected by the substituent and its position on the N3 aromatic ring. The ratio of the sulfoxide and sulfone products was quantified by isolating the products by liquid chromatography.
\end{abstract}

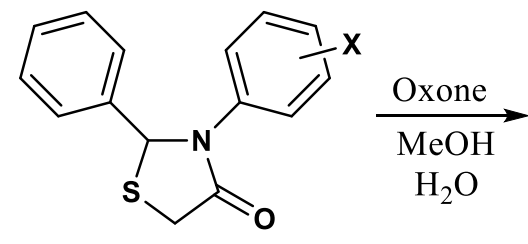

thiazolidin-4-one<smiles>O=C1CS(=O)C(c2ccccc2)N1c1ccccc1</smiles>

sulfoxide<smiles>[X]c1ccc(N2C(=O)CS(=O)(=O)C2c2ccccc2)cc1</smiles>

sulfone

Keywords: thiazolidin-4-ones, oxone, sulfoxide, sulfone

\section{Introduction}

1,3-Thiazolidin-4-ones, also known as thiazolidin-4-ones, are known to have a very wide range of biological activity. (Suryawanshi et al., 2017) (Kaushal \& Kaur, 2016) (Kumar, Kumar, Mundlia, Pradhan \& Malik, 2015) (Tripathi et al., 2014) (Jain, Vaidya, Ravichandran, Kashaw, \& Agrawal, 2012) (Abhinit, Ghodke \& Pratima, 2009) (Hamama, Ismail, Shaaban \& Zoorob, 2008) (Singh, Parmar, Raman, Virgil \& Stenberg, 1981) (Brown, 1961), so much that some have referred to it as a "magic moiety" or "wonder nucleus"(Jain et al., 2012). The $S$-oxides may show enhanced activity; for example, Miller and coworkers converted one 4-thiazolidinone to its sulfoxide and sulfone and reported that the oxides showed greater activity against some cancer cell lines than the sulfide. (Gududuru, Hurh, Dalton \& Miller, 2004) Thiazolidin-4-ones have been oxidized to sulfoxides with peracetic acid (Surrey, 1967), $\mathrm{Na}_{5} \mathrm{IO}_{6}($ Smith, Lee \& Cragoe, 1977), chloramine T (Omar, El-Kharmy \& Sharif, 1981), $\mathrm{NaIO}_{4}$ (Lee, Yergatian, Crowther \& Downie, 1990), Oxone ${ }^{\circledR}$ (one example) (Rozwadowska, Sulima \& Gzella, 2002), and $m$-CPBA (Rozwadowska \& Sulima, 2003). Oxidation from sulfide to sulfoxide makes the sulfur a chiral center, and produces cis and trans diastereomers with relation to C-2. (Rozwadowska et al., 2002) (Colombo et al., 2008). The stereocenters, however, may be configurationally unstable. (Rozwadowska et al., 2002) Oxidation of thiazolidin-4-ones to sulfones has been accomplished with $\mathrm{H}_{2} \mathrm{O}_{2} / \mathrm{Ac}_{2} \mathrm{O} / \mathrm{AcOH}$ (Troutman \& Long, 1948), and $\mathrm{KMnO}_{4}$ (Surrey, 1948).

Oxone ${ }^{\circledR}$, a mixture of potassium sulfates $\left(2 \mathrm{KHSO}_{5} / 1 \mathrm{~K}_{2} \mathrm{SO}_{4} / 1 \mathrm{KHSO}_{4}\right)$, is a very desirable material to use because it is a "green" reagent which is inexpensive, safe, and easy to use. (Yu et al., 2012) (Hussain, Green \& Ahmed, 2013) It has been used as a chemoselective reagent for the oxidation of sulfides to either sulfoxides (Trost \& Curran, 1981) (Yu et al., 2012) (Webb, 1994) (Madesclaire, 1986) or sulfones (Trost \& Curran, 1981)(Yu et al., 2012) (Webb, 1994). Selectivity toward the sulfoxide or sulfone has been shown to depend on the amount of Oxone ${ }^{\circledR}$ used, the temperature, and the solvent (Trost \& Curran, 1981) (Yu et al., 2012) (Webb, 1994). 
Although there are ample examples of Oxone ${ }^{\circledR}$-based oxidations of sulfides, there is little data related specifically to the oxidation of thiazolidin-4-ones. Rozwadowska et al., (2002) reported a single example of oxidation of a thiazolidin-4-one to its sulfoxide with this reagent. Convenient synthetic access to thiazolidin-4-one $S$-oxides would encourage further biological and pharmaceutical evaluation of these compounds, which prompted our current investigation. We have previously reported the reaction of ortho-substituted 2-aryl-3-cyclohexyl-1,3-thiazolidin-4-ones with Oxone ${ }^{\circledR}$.(Cannon et al., 2015) For all ten compounds previously evaluated, selective oxidation to the sulfoxide was realized using 3 equivalents of $O x \mathrm{x}^{\circledR}{ }^{\circledR}$ at room temperature, and subsequent oxidation to the sulfone was not observed under these reaction conditions after 25 hours. Alternatively, the sulfones were prepared with variable selectively at higher temperature (refluxing aqueous methanol) by increasing the equivalents of Oxone ${ }^{\circledR}$ used to 8.(Scheme 1) The extent of this selectivity was affected by the substituent on the $\mathrm{C} 2$ aromatic ring; sulfones were produced exclusively when the substituent (X) was $\mathrm{OCH}_{3}, \mathrm{OCH}_{2} \mathrm{CH}_{3}$, or $\mathrm{NO}_{2}$. Sulfone formation was significantly favored when the substituent was $\mathrm{CH}_{3}$ or $\mathrm{H}$. Slight preference for sulfone versus sulfoxide formation was observed when the substituent (X) was $\mathrm{CF}_{3}$, but preference for sulfoxide formation was observed for halide substituents ( $\mathrm{X}=\mathrm{F}$ or $\mathrm{Br}$ ). No clear pattern of reactivity was realized based on the substituents' electronic properties or size.<smiles>[X]c1ccccc1C1SCC(=O)N1C1CCCCC1</smiles>

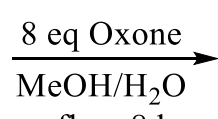

reflux, $8 \mathrm{~h}$<smiles>[X]c1ccccc1C1N(C2CCCCC2)C(=O)CS1=O</smiles><smiles>[X]c1ccccc1C1N(C2CCCCC2)C(=O)CS1(=O)=O</smiles>

$$
\mathrm{X}=\mathrm{H}, \mathrm{F}, \mathrm{Cl}, \mathrm{Br}, \mathrm{CH}_{3}, \mathrm{CF}_{3}, \mathrm{OCH}_{3}, \mathrm{OCH}_{2} \mathrm{CH}_{3}, \mathrm{NO}_{2}, 1 \text {-naphthyl }
$$

Scheme 1. Oxidation of thiazolidin-4-ones using high temperature Oxone ${ }^{\circledR}$-based reaction conditions

In this study, we report the high temperature oxidation of a series of meta- and para-substituted 3-aryl-2-phenyl-1,3-thiazolidin-4-ones with Oxone ${ }^{\circledR}$ to determine if 1) substituents on the N3 aromatic ring affect oxidative selectivity and 2) compare the substituent effects on the N3 aromatic ring to those previously reported on the $\mathrm{C} 2$ aromatic ring. We also report room temperature oxidations of this series of thiazolidin-4-ones with Oxone ${ }^{\circledR}$ and $\mathrm{KMnO}_{4}$ to ascertain the scope and selectivity of the Oxone ${ }^{\circledR}$ oxidations.

\section{Results and Discussion}

\subsection{Preparation of Meta- and Para-Substituted Thiazolidin-4-Ones}

The meta- and para-substituted 3-aryl-2-phenyl-1,3-thiazolidin-4-ones 1c-o used in this evaluation had been previously prepared by sequential condensation reactions.(Tierney et al., 2005) The meta- and para- $\mathrm{CF}_{3}$-substituted compounds, $\mathbf{1 a}$ and $\mathbf{1 b}$, were likewise prepared by condensation of benzaldehyde with the respective substituted aniline to produce an imine intermediate, followed by condensation with thioglycolic acid. (Scheme 2) Reaction progress in both steps was monitored by the collection of water in a Dean-Stark trap. 


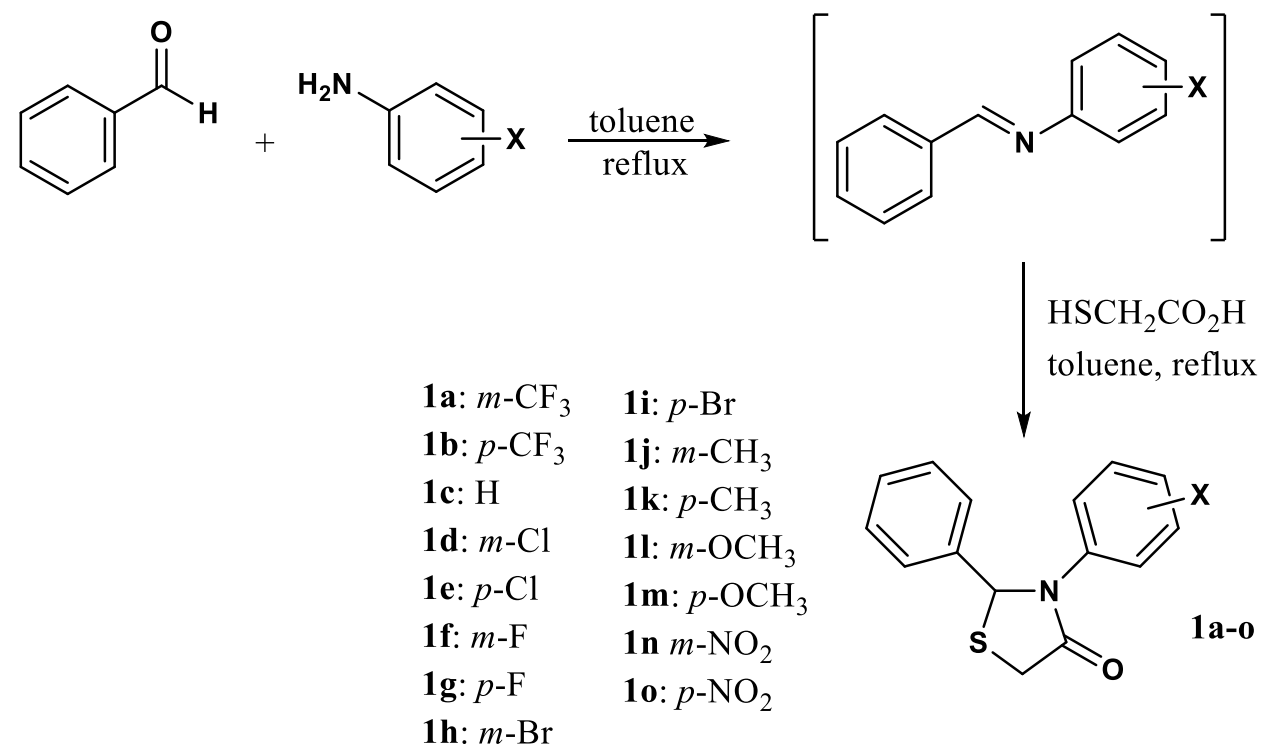

Scheme 2. Synthesis of meta- and para-substituted 3-aryl-2-phenyl-1,3-thiazolidin-4-ones 1a-o

\subsection{Low Temperature Oxone ${ }^{\circledR}$ Oxidations of Thiazolidin-4-Ones}

Exclusive formation of sulfoxide compounds $\mathbf{2 a - 2 o}$ was realized by performing the oxidation at room temperature with a 3 equivalents of Oxone ${ }^{\circledR}$. (Scheme 3) The reaction time for sulfoxide formation was optimized at $1 \mathrm{~h}$ to insure complete conversion of the thiazolidin-4-ones which was followed by thin layer chromatography (TLC). Results are summarized in Table 1. Oxidation of $\mathbf{1 e}$ at extended reaction times showed no sulfone formation by ${ }^{1} \mathrm{H}$ NMR at periods as long as $25 \mathrm{~h}$. Clearly, low temperature oxidation with a reduced number of Oxone ${ }^{\circledR}$ equivalents favors sulfoxide formation as was previously observed for the 3-cyclohexyl-thiazolidin-4-ones (Cannon et al., 2015).

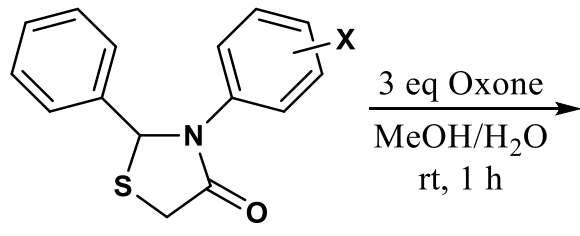

1a-o<smiles>[X]c1ccc(N2C(=O)CS(=O)C2c2ccccc2)cc1</smiles>

2a-o

Scheme 3. Selective low temperature Oxone ${ }^{\circledR}$ oxidation of thiazolidin-4-ones to sulfoxides

Table 1. Meta- and para-substituted 3-aryl-2-phenyl-1,3-thiazolidin-4-one sulfoxides 2a-2o synthesized according to Scheme 4

\begin{tabular}{|c|c|c|c|}
\hline Product & $\%$ Yield & Melting Point $\left({ }^{\circ} \mathrm{C}\right)$ & $\begin{array}{c}\mathrm{R}_{\mathrm{f}} \\
\text { (3:1 cyclohexane:EtOAc) }\end{array}$ \\
\hline $\mathbf{2 a}, \mathrm{X}=m-\mathrm{CF}_{3}$ & 98 & $64-65$ & 0.066 \\
\hline $\mathbf{2 b}, \mathrm{X}=p-\mathrm{CF}_{3}$ & 94 & $182-183$ & 0.138 \\
\hline 2c, $X=H$ & 96 & $178-180$ & 0.159 \\
\hline 2d, $\mathrm{X}=m-\mathrm{Cl}$ & 74 & $166-168$ & 0.160 \\
\hline $2 \mathbf{e}, \mathrm{X}=p-\mathrm{Cl}$ & 98 & $164-165$ & 0.094 \\
\hline 2f, $\mathrm{X}=m-\mathrm{F}$ & 99 & $149-152$ & 0.137 \\
\hline $2 \mathrm{~g}, \mathrm{X}=p-\mathrm{F}$ & 99 & $188-190$ & 0.230 \\
\hline $\mathbf{2 h}, \mathrm{X}=m-\mathrm{Br}$ & 75 & $167-169$ & 0.112 \\
\hline $2 \mathbf{i}, \mathrm{X}=p-\mathrm{Br}$ & 99 & $190-191$ & 0.134 \\
\hline $2 \mathbf{j}, \mathrm{X}=m-\mathrm{CH}_{3}$ & 97 & $161-162$ & 0.143 \\
\hline $\mathbf{2 k}, \mathrm{X}=p-\mathrm{CH}_{3}$ & 97 & $179-180$ & 0.157 \\
\hline 2l, $\mathrm{X}=m-\mathrm{OCH}_{3}$ & 99 & d 219 & 0.56 \\
\hline $\mathbf{2 m}, \mathrm{X}=p-\mathrm{OCH}_{3}$ & 99 & $188-189$ & 0.101 \\
\hline 2n, $\mathrm{X}=m-\mathrm{NO}_{2}$ & 99 & $205-207$ & 0.089 \\
\hline 2o, $\mathrm{X}=p-\mathrm{NO}_{2}$ & 44 & $\mathrm{~d} 215$ & 0.075 \\
\hline
\end{tabular}


Room temperature oxidations with 8 equivalents of Oxone ${ }^{\circledR}$ were performed with compounds 1c and 1e.(Scheme 4) Although increasing both equivalents of Oxone ${ }^{\circledR}$ and reaction time did result in the formation of sulfones, yields were low and sulfoxide products were still significantly favored.

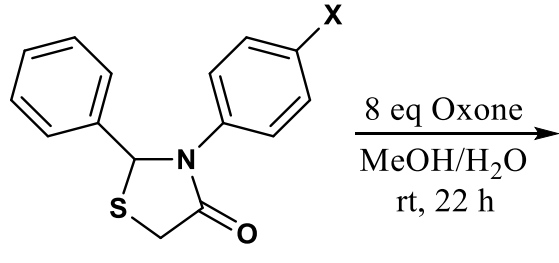

1c, $\mathrm{X}=\mathrm{H}$

1e, $\mathrm{X}=\mathrm{Cl}$

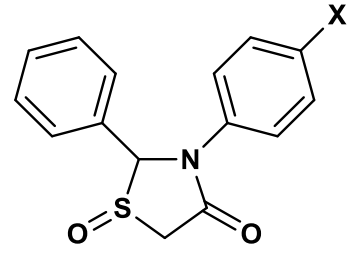

$2 \mathbf{c}(80 \%)$

$2 \mathrm{e}(67 \%)$<smiles>[X]c1ccc(N2C(=O)CS(=O)(=O)C2c2ccccc2)cc1</smiles>

$3 \mathbf{c}(9 \%)$

3e $(8 \%)$

Scheme 4. Low temperature Oxone ${ }^{\circledR}$ oxidation using 8 equivalents of Oxone ${ }^{\circledR}$

\subsection{High Temperature Oxone ${ }^{\circledR}$ Oxidations of Thiazolidin-4-Ones}

Compounds 1a-o were oxidized according to the high temperature Oxone ${ }^{\circledR}$-based reaction conditions that had been previously optimized for 2-aryl-3-cyclohexyl-1,3-thiazolidin-4-ones (Cannon et al., 2015) to determine if selective formation of sulfones could be realized according to the substituted N3 aromatic ring (Scheme 5). Results are presented in Table 2.

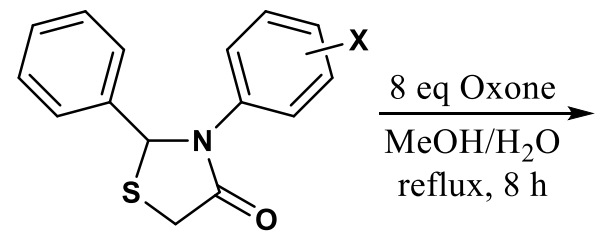

1a-o<smiles>[X]c1ccc(N2C(=O)CS(=O)C2c2ccccc2)cc1</smiles>

2a-o

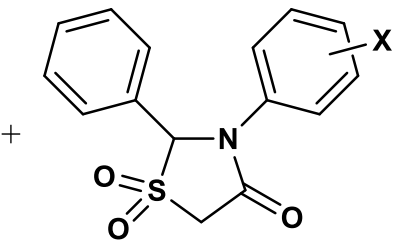

3a-o

Scheme 5. High temperature Oxone ${ }^{\circledR}$ oxidation of thiazolidin-4-ones

The ratio indicates the relative amounts of sulfoxide to sulfone isolated by chromatography.

Table 2. Oxidation of thiazolidin-4-ones 1a-o using high temperature Oxone ${ }^{\circledR}$-based reaction conditions

\begin{tabular}{lcc}
\hline Thiazolidin-4-one (1) & Sulfoxide:Sulfone (2:3) & Total Yield \\
\hline 1a, $\mathrm{X}=m-\mathrm{CF}_{3}$ & $1.0: 2.9$ & $43 \%$ \\
$\mathbf{1 b}, \mathrm{X}=p-\mathrm{CF}_{3}$ & $1.0: 2.0$ & $40 \%$ \\
$\mathbf{1 c}, \mathrm{X}=\mathrm{H}$ & $1.0: 3.2$ & $66 \%$ \\
$\mathbf{1 d}, \mathrm{X}=m-\mathrm{Cl}$ & $1.0: 2.0$ & $67 \%$ \\
$\mathbf{1 e}, \mathrm{X}=p-\mathrm{Cl}$ & $1.0: 1.7$ & $58 \%$ \\
$\mathbf{1 f}, \mathrm{X}=m-\mathrm{F}$ & $1.0: 1.6$ & $71 \%$ \\
$\mathbf{1 g}, \mathrm{X}=p-\mathrm{F}$ & $1.0: 1.5$ & $94 \%$ \\
$\mathbf{1 h}, \mathrm{X}=m-\mathrm{Br}$ & $1.0: 2.6$ & $56 \%$ \\
$\mathbf{1 i}, \mathrm{X}=p-\mathrm{Br}$ & $1.0: 1.9$ & $54 \%$ \\
$\mathbf{1 j}, \mathrm{X}=m-\mathrm{CH}_{3}$ & $1.1: 1.0$ & $65 \%$ \\
$\mathbf{1 k}, \mathrm{X}=p-\mathrm{CH}_{3}$ & $1.0: 1.5$ & $55 \%$ \\
$\mathbf{1 1}, \mathrm{X}=m-\mathrm{OCH}_{3}$ & sulfoxide only & $14 \%$ \\
$\mathbf{1 m}, \mathrm{X}=p-\mathrm{OCH}_{3}$ & $1.0: 12.3$ & $78 \%$ \\
$\mathbf{1 n}, \mathrm{X}=m-\mathrm{NO}_{2}$ & $1.0: 7.4$ & $61 \%$ \\
$\mathbf{1 0}, \mathrm{X}=p-\mathrm{NO}_{2}$ & $1.0: 6.0$ & $48 \%$ \\
\hline
\end{tabular}

Results in Table 2 show that sulfone formation varied according to both the substituent and its position on the N3 aromatic ring. However, few clear substitution/reactivity correlations can be ascertained. The selectivity of sulfone versus sulfoxide formation was favored when electron donating substituents $\left(\mathrm{X}=\mathrm{CH}_{3}\right.$ and $\left.\mathrm{OCH}_{3}\right)$ were in the para position; for all other substituents, meta substitution showed higher sulfone selectivity versus para substitution. Oxidation yields showed no correlation with substituent location: in two sets $\left(\mathrm{X}=\mathrm{F}\right.$ and $\left.\mathrm{OCH}_{3}\right)$ the para-substituted compounds demonstrated higher yields, and in the remaining five sets the meta-substituted compounds demonstrated higher yields.

The effect of the substituents' electronic properties on yield somewhat mirrored previously reported oxidations of the 2-aryl-3-cyclohexyl-1,3-thiazolidin-4-ones with Oxone ${ }^{\circledR}$, which also showed the highest sulfone selectivity for $\mathrm{OCH}_{3}$ 
and $\mathrm{NO}_{2}$ substituents (Cannon et al., 2015). The highest sulfone selectivity was observed for $p-\mathrm{OCH}_{3}$ substituted compound 1m (>12:1 sulfone:sulfoxide). High sulfone selectivity was also realized for both meta- and para- $\mathrm{NO}_{2}$ substituted compounds $1 \mathbf{n}$ and 1o, respectively, and reasonable selectivity was seen for $\mathrm{X}=\mathrm{H}(\mathbf{1 c})$. However, there are significant differences between the 3-aryl-2-phenyl-1,3-thiazolidin-4-ones and

2-aryl-3-cyclohexyl-1,3-thiazolidin-4-ones as well. Higher sulfone selectivity was observed for all the halide- and $\mathrm{CF}_{3}$-substituted 3-aryl-thiazolidin-4-ones versus the 3-cyclohexyl-thiazolidin-4-ones, while sulfone selectivity dropped dramatically for the $\mathrm{CH}_{3}$-substituted 3-aryl-thiazolidin-4-ones. Thus, although the strongly electron donating $p$ - $\mathrm{OCH}_{3}$ and the strongly electron withdrawing $\mathrm{NO}_{2}$ substituents still showed the best sulfone selectivity, all other substituents on the N3 aromatic ring demonstrated less variation amongst each other than was seen in the previous study with substituents on the $\mathrm{C} 2$ aromatic ring. This decreased variation is probably rooted in the more remote location of the $\mathrm{N} 3$ aromatic ring from the sulfur, which is expected to reduce the corresponding electronic effect of substituents on the N3 aromatic ring.

Overall, the total oxidation yields are lower for the 3-aryl-2-phenyl-1,3-thiazolidin-4-ones (average yield $=58 \%$ ) versus 2-aryl-3-cyclohexyl-1,3-thiazolidin-4-ones (average yield $=89 \%$ ) despite reactant conversions of $100 \%$ for all compounds evaluated. To determine if sulfones were stable under reaction conditions, isolated sulfone $\mathbf{3 b}\left(p-\mathrm{CF}_{3}\right)$ was subjected to 8 Oxone ${ }^{\circledR}$ equivalents in refluxing aqueous methanol for $8 \mathrm{~h}$. No significant decomposition was detected by TLC, and 3b was recovered quantitatively. Therefore, product decomposition cannot account for the observed lower yields.

\section{4 $\mathrm{KMnO}_{4}$-Based Oxidations of Thiazolidin-4-Ones}

Since exclusive formation of 3-aryl-2-phenyl-1,3-thiazolidin-4-one sulfones was not reliably realized using Oxone ${ }^{\circledR}$, an alternative syntheses of the sulfones using aqueous $\mathrm{KMnO}_{4}$ was evaluated.(Surrey, 1948) This method was applied to compounds 1a-1o to produce the corresponding sulfones (Scheme 6); results are summarized in Table 3. Although no measures were taken to optimize this reaction, the oxidation yields are overall significantly higher for the 3-aryl-2-phenyl-1,3-thiazolidin-4-ones (average yield=76\%) versus 2-aryl-3-cyclohexyl-1,3-thiazolidin-4-ones (average yield $=56 \%$ ) previously synthesized by this method (Cannon et al., 2015).

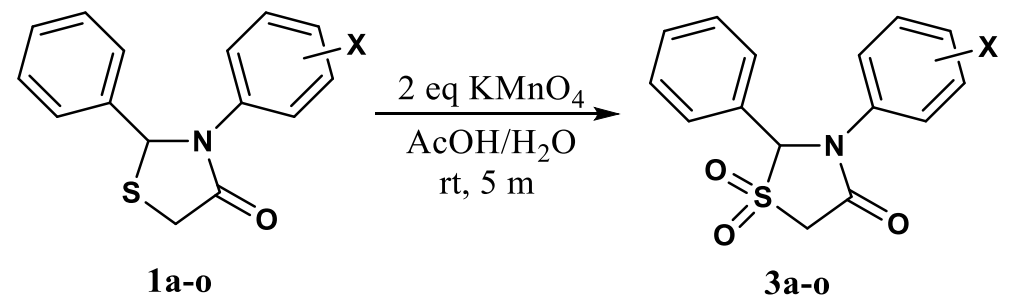

Scheme 6. $\mathrm{KMnO}_{4}$ oxidation of thiazolidin-4-ones to sulfones

Table 3. Meta- and para-substituted 3-aryl-2-phenyl-1,3-thiazolidin-4-one sulfones 3a-3o synthesized according to Scheme 6

\section{Conclusion}

\begin{tabular}{lcc}
\hline \multicolumn{1}{c}{ Product } & \% Yield & Melting Point $\left({ }^{\circ} \mathrm{C}\right)$ \\
\hline 3a, $\mathrm{X}=m-\mathrm{CF}_{3}$ & 78 & $106-110$ \\
$\mathbf{3 b}, \mathrm{X}=p-\mathrm{CF}_{3}$ & 81 & $186-187$ \\
$\mathbf{3 c}, \mathrm{X}=\mathrm{H}$ & 76 & $178-180$ \\
$\mathbf{3 d}, \mathrm{X}=m-\mathrm{Cl}$ & 82 & $183-184$ \\
$\mathbf{3 e}, \mathrm{X}=p-\mathrm{Cl}$ & 76 & $176-178$ \\
$\mathbf{3 f}, \mathrm{X}=m-\mathrm{F}$ & 77 & $171-172$ \\
$\mathbf{3 g}, \mathrm{X}=p-\mathrm{F}$ & 56 & $141-142$ \\
$\mathbf{2 h}, \mathrm{X}=m-\mathrm{Br}$ & 77 & $190-191$ \\
$\mathbf{2 i}, \mathrm{X}=p-\mathrm{Br}$ & 76 & $201-204$ \\
$\mathbf{2 j}, \mathrm{X}=m-\mathrm{CH}_{3}$ & 65 & $173-175$ \\
$\mathbf{2 k}, \mathrm{X}=p-\mathrm{CH}_{3}$ & 78 & $104-105$ \\
$\mathbf{2 l}, \mathrm{X}=m-\mathrm{OCH}_{3}$ & 83 & $214-217$ \\
$\mathbf{2 m}, \mathrm{X}=p-\mathrm{OCH}_{3}$ & 95 & $104-105$ \\
$\mathbf{2 n}, \mathrm{X}=m-\mathrm{NO}_{2}$ & 80 & $199-200$ \\
$\mathbf{2 o}, \mathrm{X}=p-\mathrm{NO}_{2}$ & 56 & $204-206$ \\
\hline
\end{tabular}

$S$-oxidation of 3-aryl-2-phenyl-1,3-thiazolidin-4-ones with Oxone ${ }^{\circledR}$ was dependent on the reaction temperature, equivalents of Oxone ${ }^{\circledR}$ used, the substituent, and its location on the N3 aromatic ring. For all thiazolidin-4-ones 
evaluated in this study, selective oxidation to the sulfoxide was realized by using 3 equivalents of Oxone ${ }^{\circledR}$ at room temperature. By increasing the amount of Oxone $^{\circledR}$ to 8 equivalents, room temperature oxidation of two thiazolidin-4-ones (1c and 1e) resulted in the formation of sulfone in yields $<10 \%$; sulfoxide formation was still preferred. At high temperature using 8 equivalents of Oxone ${ }^{\circledR}$, the extent of selectivity in sulfone formation was affected by the substituent and its location on the N3 aromatic ring. The only clear substituent/reactivity correlation evidenced was better selectivity of sulfone versus sulfoxide formation when electron donating substituents $\left(\mathrm{X}=\mathrm{CH}_{3}\right.$ and $\left.\mathrm{OCH}_{3}\right)$ were in the para position; for all other substituents, meta substitution showed higher sulfone selectivity versus para substitution. Unlike the 3-cyclohexyl-2-phenyl-1,3-thiazolidin-4-ones, exclusive formation of sulfone versus sulfoxide was never realized by Oxone ${ }^{\circledR}$ oxidation. Exclusive sulfone formation was best achieved using 2 equivalents of $\mathrm{KMnO}_{4}$.

\section{Experimental}

Reagent chemicals were obtained from commercial suppliers; Oxone ${ }^{\circledR}$ was purchased from Aldrich Chemical Company. TLC and chromatography plates (silica gel GF, 250 micron) were purchased from Analtech. Reagent grade solvents were used without further purification.

Most spectra were recorded on a Bruker 300 at $298 \mathrm{~K}$ observing ${ }^{1} \mathrm{H}$ and ${ }^{13} \mathrm{C}$ at 300.15 and $75.48 \mathrm{MHz}$, respectively. These samples were dissolved in $\mathrm{CDCl}_{3}$ at a concentration of $100 \mathrm{mg} / \mathrm{mL}$ using precision bore $5 \mathrm{~mm}$ NMR tubes supplied by Norell, Inc. The spectrometer was locked to either the deuterium or carbon resonance of $\mathrm{CDCl}_{3}$ and all chemical shifts were referenced to residual $\mathrm{CHCl}_{3}$.

Infrared spectra were obtained as an evaporated thin film on a sodium chloride plate (Janos Technology, Inc) on a Nicolet Nexus 670 spectrometer using 32 scans at a $2 \mathrm{~cm}^{-1}$ resolution. Mass spectra were recorded on a Varian $2100 \mathrm{G}$ ion trap mass spectrometer, fitted with a Varian 3900 gas chromatograph: column - Factor 4 VF-5ms $0.25 \mathrm{~mm}$ id, $30 \mathrm{~m}$, $0.25 \mu \mathrm{m}$ film thickness, He carrier gas, $1.0 \mathrm{ml} / \mathrm{min}$ flow, $80^{\circ} \mathrm{C}$ for 1 minute isothermal $15^{\circ} \mathrm{C} / \mathrm{min}$ to $275^{\circ} \mathrm{C}$ then $275^{\circ} \mathrm{C}$ for 3 minutes isothermal, injector temp $250^{\circ} \mathrm{C}, 0 \mathrm{~min}, 1: 50$ split. Melting points are uncorrected; a Uni-Melt capillary melting point apparatus was used.

\subsection{Preparation of 3-Aryl-Substituted -2-Phenyl-Thiazolidin-4-Ones}

All thiazolidine-4-ones used in this study except the $\mathrm{CF}_{3}$-substituted compounds had been previously synthesized and characterized. Thiazolidine-4-ones were prepared using the procedure previously described. (Tierney et al., 2005) Isolated yields of the $\mathrm{CF}_{3}$-substituted thiazolidine-4-ones are based on the corresponding aniline as the limiting reactant. No attempt was made to maximize the product yields. The $p-\mathrm{CF}_{3}$ compound $\mathbf{1 b}$ was purified by recrystallization using methanol. The $m-\mathrm{CF}_{3}$ compound 1a, a colorless oil, was purified chromatography on pre-coated plates of silica gel GF $250 \mu \mathrm{m}$ using 3:1 cyclohexane/EtOAc.

2-phenyl-2-(3-trifluoromethylphenyl)-1,3-thiazolidin-4-one (1a). Yield: $(98 \%)$; $\mathrm{R}_{\mathrm{f}}=0.607$; $\mathrm{IR}$ : $\mathrm{cm}^{-1} 1687.6(\mathrm{C}=\mathrm{O})$; ${ }^{1} \mathrm{H}$ NMR $\left(\mathrm{CDCl}_{3}\right)$ : 7.42-7.14 (9H, aromatics), $6.04(1 \mathrm{H}, \mathrm{s}, \mathrm{C} 2), 3.89(1 \mathrm{H}, \mathrm{dd}, \mathrm{C} 5, \mathrm{~J}=1.3 \mathrm{~Hz}$, and J = 15.8 Hz), $3.77(1 \mathrm{H}$, $\mathrm{d}, \mathrm{C} 5, \mathrm{~J}=15.8 \mathrm{~Hz}) ;{ }^{13} \mathrm{C}$ NMR: $171.29(\mathrm{C} 4), 138.62,138.21,131.51(\mathrm{q}, \mathrm{J}=32.8 \mathrm{~Hz}), 129.73,129.34,129.20,128.78$, 127.04, 123.90 (q, J = 272.8.0 Hz), 123.68 (q, J = 3.4 Hz), 122.22 (q, J = 4.0 Hz), $65.42(\mathrm{C} 2), 33.52$ (C5); MS: (m/z) $324\left([\mathrm{M}+\mathrm{H}]^{+}\right), \mathrm{C}_{16} \mathrm{H}_{13} \mathrm{ONSF}_{3}(324.07)$.

2-phenyl-2-(4-trifluoromethylphenyl)-1,3-thiazolidin-4-one (1b). Yield: $(94 \%) ; \mathrm{R}_{\mathrm{f}}=0.586 ;$ m.p. 107-108 ${ }^{\circ} \mathrm{C}$; IR: $\mathrm{cm}^{-1} 1681.4(\mathrm{C}=\mathrm{O}) ;{ }^{1} \mathrm{H}$ NMR $\left(\mathrm{CDCl}_{3}\right): 7.46-7.17(9 \mathrm{H}$, aromatics), $6.17(1 \mathrm{H}, \mathrm{s}, \mathrm{C} 2), 3.99(1 \mathrm{H}, \mathrm{dd}, \mathrm{C} 5, \mathrm{~J}=1.4 \mathrm{~Hz}$, and $\mathrm{J}$ $=16.1 \mathrm{~Hz}), 3.86(1 \mathrm{H}, \mathrm{d}, \mathrm{C} 5, \mathrm{~J}=16.4 \mathrm{~Hz}) ;{ }^{13} \mathrm{C}$ NMR: $171.38(\mathrm{C} 4), 140.87,139.04,129.30,128.56(\mathrm{q}, \mathrm{J}=32.8 \mathrm{~Hz})$, 126.76, $126.45(\mathrm{q}, \mathrm{J}=4.1 \mathrm{~Hz}), 124.95,123.86(\mathrm{q}, \mathrm{J}=272.4 \mathrm{~Hz}), 65.19(\mathrm{C} 2), 33.56(\mathrm{C} 5) ; \mathrm{MS}:(\mathrm{m} / \mathrm{z}) 324\left([\mathrm{M}+\mathrm{H}]^{+}\right)$, $\mathrm{C}_{16} \mathrm{H}_{13} \mathrm{ONSF}_{3}$ (324.07).

\subsection{General Procedure for the RT Synthesis of Thiazolidin-4-One Sulfoxides via Oxone ${ }^{\circledR}$}

Thiazolidin-4-one $(1.01 \mathrm{mmol})$ was typically dissolved in methanol $(8.0 \mathrm{~mL})$, to which an aqueous solution of Oxone ${ }^{\circledR}$ (461 mg, $3.03 \mathrm{mmol}$ calculated as $\mathrm{KHSO}_{5}, 152.2 \mathrm{~g} \mathrm{~mol}^{-1}$, in $4.0 \mathrm{~mL}$ water) was added dropwise at room temperature with vigorous stirring. After the addition, the reaction mixture was stirred for $1 \mathrm{~h}$. Water $(40 \mathrm{~mL})$ was then added to the mixture to dissolve precipitates, and the mixture was extracted with $\mathrm{CHCl}_{3}(3 \times 15 \mathrm{~mL})$. The combined $\mathrm{CHCl}_{3}$ layers were dried with $\mathrm{Na}_{2} \mathrm{SO}_{4}$, and the $\mathrm{CHCl}_{3}$ was removed in vacuo followed by chromatography. Except for compounds $\mathbf{2 k}$ $(p-\mathrm{Me})$ and $2 \mathrm{o}\left(p-\mathrm{NO}_{2}\right)$, two sets of diastereomeric isomers were evident by ${ }^{1} \mathrm{H}$ NMR and ratios were calculated by integration of the hydrogens on $\mathrm{C} 2$.

Sulfoxide-2-phenyl-2-(3-trifluoromethylphenyl)-1,3-thiazolidin-4-one (2a). Yield: (98\%); $\mathrm{R}_{\mathrm{f}}=0.066$; m.p. $64-65^{\circ} \mathrm{C}$; IR cm ${ }^{-1} 1703.9(\mathrm{C}=\mathrm{O}), 1061.0(\mathrm{~S}=\mathrm{O}) ;{ }^{1} \mathrm{H}$ NMR $\left(\mathrm{CDCl}_{3}\right), 7.58-7.32(9 \mathrm{H}$, aromatics). Major isomer $(95 \%), 6.03(1 \mathrm{H}, \mathrm{s}$, $\mathrm{C} 2), 3.87(1 \mathrm{H}, \mathrm{d}, \mathrm{C} 5, \mathrm{~J}=17.0 \mathrm{~Hz}), 3.56(1 \mathrm{H}, \mathrm{dd}, \mathrm{C} 5 \mathrm{~J}=1.3 \mathrm{~Hz}$ and $\mathrm{J}=17.1 \mathrm{~Hz})$. Minor isomer $(5 \%), 6.10(1 \mathrm{H}, \mathrm{s}, \mathrm{C} 2)$, $3.98(1 \mathrm{H}, \mathrm{d}, \mathrm{C} 5, \mathrm{~J}=17.0 \mathrm{~Hz}), 3.77$ (1H, d, C5, J = 16.4 Hz); ${ }^{13} \mathrm{C}$ NMR: 168.66 (C4), 138.69, 137.57, 131.70 (q, J = 32.7 $\mathrm{Hz}), 130.30,130.10,129.96,127.95,126.23,126.00,124.93,123.55$ (q, J = 273.5 Hz), 123.42 (q, J = 3.3 Hz), 122.20, 
119.98 (q, J = 3.6 Hz), $85.85(\mathrm{C} 2), 52.19$ (C5); MS: (m/z) $340\left([\mathrm{M}+\mathrm{H}]^{+}\right), \mathrm{C}_{16} \mathrm{H}_{13} \mathrm{O}_{2} \mathrm{NSF}_{3}(340.06)$.

Sulfoxide-2-phenyl-2-(4-trifluoromethylphenyl)-1,3-thiazolidin-4-one (2b). Yield: (94\%); $\mathrm{R}_{\mathrm{f}}=0.138 ;$ m.p. 182-183 ${ }^{\circ} \mathrm{C}$; IR cm ${ }^{-1} 1693.6(\mathrm{C}=\mathrm{O}), 1078.1(\mathrm{~S}=\mathrm{O}) ;{ }^{1} \mathrm{H}$ NMR $\left(\mathrm{CDCl}_{3}\right), 7.58-7.32(9 \mathrm{H}$, aromatics). Major isomer (93\%), 6.06 $(1 \mathrm{H}, \mathrm{s}, \mathrm{C} 2), 3.88(1 \mathrm{H}, \mathrm{d}, \mathrm{C} 5, \mathrm{~J}=17.2 \mathrm{~Hz}), 3.59(1 \mathrm{H}, \mathrm{dd}, \mathrm{C} 5 \mathrm{~J}=1.4 \mathrm{~Hz}$ and $\mathrm{J}=17.2 \mathrm{~Hz})$. Minor isomer $(7 \%), 6.11(1 \mathrm{H}$, s, C2), $4.00(1 \mathrm{H}, \mathrm{d}, \mathrm{C} 5, \mathrm{~J}=17.1 \mathrm{~Hz}), 3.81(1 \mathrm{H}, \mathrm{d}, \mathrm{C} 5, \mathrm{~J}=16.6 \mathrm{~Hz}) ;{ }^{13} \mathrm{C}$ NMR: $168.69(\mathrm{C} 4), 141.30,130.39,130.21$, 130.13, 128.43 (q, J = 33.5 Hz), 126.74, $126.54(\mathrm{q}, \mathrm{J}=4.3 \mathrm{~Hz}), 125.05(\mathrm{~d}, 18.6 \mathrm{~Hz}), 124.60,123.79$ (q, J = 271.5 Hz), 122.60, 122.44, 85.80 (C2), 52.33 (C5); MS: (m/z) $340\left([\mathrm{M}+\mathrm{H}]^{+}\right), \mathrm{C}_{16} \mathrm{H}_{13} \mathrm{O}_{2} \mathrm{NSF}_{3}$ (340.06).

Sulfoxide-2,3-diphenyl-1,3-thiazolidin-4-one (2c). Yield (96\%); $\mathrm{R}_{\mathrm{f}}=0.159$; m.p. 163-164 ${ }^{\circ} \mathrm{C}: \mathrm{IR}: \mathrm{cm}^{-1} 1681.8(\mathrm{C}=\mathrm{O})$, $1050.5(\mathrm{~S}=\mathrm{O}) ;{ }^{1} \mathrm{H}$ NMR $\left(\mathrm{CDCl}_{3}\right): 7.50-7.21(10 \mathrm{H}$, aromatics). Major isomer $(98 \%), 5.97(1 \mathrm{H}, \mathrm{s}, \mathrm{C} 2), 3.90(1 \mathrm{H}, \mathrm{d}, \mathrm{C}$, $\mathrm{J}=17.0 \mathrm{~Hz}), 3.58(1 \mathrm{H}, \mathrm{dd}, \mathrm{C} 5 \mathrm{~J}=1.2 \mathrm{~Hz}$ and $\mathrm{J}=16.9 \mathrm{~Hz})$. Minor isomer $(2 \%), 6.07(1 \mathrm{H}, \mathrm{s}, \mathrm{C} 2), 3.98(1 \mathrm{H}, \mathrm{d}, \mathrm{C} 5, \mathrm{~J}=$ $16.6 \mathrm{~Hz}), 3.79(1 \mathrm{H}, \mathrm{d}, \mathrm{C} 5, \mathrm{~J}=16.6 \mathrm{~Hz}) ;{ }^{13} \mathrm{C}$ NMR: $168.41(\mathrm{C} 4), 138.04,130.73,130.03,129.92,129.36,127.01,126.38$, 123.50, 86.41 (C2), 52.28 (C5); MS: (m/z) $272\left([\mathrm{M}+\mathrm{H}]^{+}\right), \mathrm{C}_{15} \mathrm{H}_{14} \mathrm{O}_{2} \mathrm{NS}(272.07)$.

Sulfoxide-3-(3-chlorophenyl)-2-phenyl-1,3-thiazolidin-4-one (2d). Yield (74\%); $\mathrm{R}_{\mathrm{f}}=0.160 ;$ m.p. 166-168 ${ }^{\circ} \mathrm{C}$; IR: $\mathrm{cm}^{-1} 1685.4(\mathrm{C}=\mathrm{O}), 1053.3(\mathrm{~S}=\mathrm{O}) ;{ }^{1} \mathrm{H}$ NMR $\left(\mathrm{CDCl}_{3}\right): 7.48-7.09(9 \mathrm{H}$, aromatics). Major isomer $(94 \%), 5.92(1 \mathrm{H}, \mathrm{s}$, $\mathrm{C} 2), 3.80(1 \mathrm{H}, \mathrm{d}, \mathrm{C} 5, \mathrm{~J}=17.2 \mathrm{~Hz}), 3.49(1 \mathrm{H}, \mathrm{dd}, \mathrm{C} 5 \mathrm{~J}=1.3 \mathrm{~Hz}$ and $\mathrm{J}=17.2 \mathrm{~Hz})$. Minor isomer $(6 \%), 6.00(1 \mathrm{H}, \mathrm{s}, \mathrm{C} 2)$, $3.92(1 \mathrm{H}, \mathrm{d}, \mathrm{C} 5, \mathrm{~J}=16.5 \mathrm{~Hz}), 3.70(1 \mathrm{H}, \mathrm{d}, \mathrm{C} 5, \mathrm{~J}=16.5 \mathrm{~Hz}) ;{ }^{13} \mathrm{C}$ NMR: $168.49(\mathrm{C} 4), 167.78(\mathrm{C} 4), 139.21,138.09$, $134.90,134.47,130.30,130.20,130.05,129.89,129.02,128.93,126.99,126.25,124.99,123.36,122.75,121.03,85.97$ (C2), 52.21 (C5); MS: (m/z) $306\left([\mathrm{M}+\mathrm{H}]^{+}\right), \mathrm{C}_{15} \mathrm{H}_{13} \mathrm{O}_{2} \mathrm{NSCl}(306.04)$.

Sulfoxide-3-(4-chlorophenyl)-2-phenyl-1,3-thiazolidin-4-one (2e). Yield (98\%); $\mathrm{R}_{\mathrm{f}}=0.094 ; \mathrm{m} . p .164-165{ }^{\circ} \mathrm{C}$; IR: $\mathrm{cm}^{-1} 1715.7(\mathrm{C}=\mathrm{O}), 1053.0(\mathrm{~S}=\mathrm{O}) ;{ }^{1} \mathrm{H}$ NMR $\left(\mathrm{CDCl}_{3}\right): 7.42-7.21(9 \mathrm{H}$, aromatics). Major isomer $(96 \%), 5.88(1 \mathrm{H}, \mathrm{s}$, C2), $3.82(1 \mathrm{H}, \mathrm{d}, \mathrm{C} 5, \mathrm{~J}=17.0 \mathrm{~Hz}), 3.50(1 \mathrm{H}, \mathrm{d}, \mathrm{C} 5 \mathrm{~J}=17.0 \mathrm{~Hz})$. Minor isomer $(4 \%), 5.97(1 \mathrm{H}, \mathrm{s}, \mathrm{C} 2), 3.92(1 \mathrm{H}, \mathrm{d}, \mathrm{C} 5$, $\mathrm{J}=16.7 \mathrm{~Hz}), 3.73(1 \mathrm{H}, \mathrm{d}, \mathrm{C} 5 \mathrm{~J}=17.1 \mathrm{~Hz}) ;{ }^{13} \mathrm{C}$ NMR: $168.44(\mathrm{C} 4), 136.63,132.47,130.39,130.31,130.13,129.55$, 129.24, 129.12, 129.03, 126.34, 126.02, 125.74, 124.56, 121.09, 114.70, 86.26 (C2), 52.28 (C5); MS: (m/z) 306 $\left([\mathrm{M}+\mathrm{H}]^{+}\right), \mathrm{C}_{15} \mathrm{H}_{13} \mathrm{O}_{2} \mathrm{NSCl}(306.04)$.

Sulfoxide-3-(3-fluorophenyl)-2-phenyl-1,3-thiazolidin-4-one (2f). Yield (99\%); $\mathrm{R}_{\mathrm{f}}=0.137$; m.p. 149-152 ${ }^{\circ} \mathrm{C}$; IR: $\mathrm{cm}^{-1}$ $1709.5(\mathrm{C}=\mathrm{O}), 1055.8(\mathrm{~S}=\mathrm{O}) ;{ }^{1} \mathrm{H}$ NMR $\left(\mathrm{CDCl}_{3}\right): 7.46-6.85(9 \mathrm{H}$, aromatics). Major isomer $(93 \%), 6.01(1 \mathrm{H}, \mathrm{s}, \mathrm{C} 2)$, $3.86(1 \mathrm{H}, \mathrm{d}, \mathrm{C} 5, \mathrm{~J}=17.4 \mathrm{~Hz}), 3.55(1 \mathrm{H}, \mathrm{dd}, \mathrm{C} 5 \mathrm{~J}=1.6 \mathrm{~Hz}$ and $\mathrm{J}=17.4 \mathrm{~Hz})$. Minor isomer $(7 \%), 6.07(1 \mathrm{H}, \mathrm{s}, \mathrm{C} 2), 3.98$ $(1 \mathrm{H}, \mathrm{d}, \mathrm{C} 5, \mathrm{~J}=16.8 \mathrm{~Hz}), 3.76(1 \mathrm{H}, \mathrm{d}, \mathrm{C} 5 \mathrm{~J}=16.6 \mathrm{~Hz}) ;{ }^{13} \mathrm{C}$ NMR: $168.60(\mathrm{C} 4), 162.86(\mathrm{~d}, \mathrm{~J}=246.4), 139.55(\mathrm{~d}, \mathrm{~J}=$ $12.8 \mathrm{~Hz}), 130.50(\mathrm{~d}, \mathrm{~J}=9.6 \mathrm{~Hz}), 130.21,130.58,128.96(\mathrm{~d}, \mathrm{~J}=12.8), 128.04,126.26,120.1(\mathrm{~J}=3.1 \mathrm{~Hz}), 118.15(\mathrm{~d}, \mathrm{~J}=$ $2.3 \mathrm{~Hz}), 113.68(\mathrm{~d}, \mathrm{~J}=21.5 \mathrm{~Hz}), 112.29(\mathrm{~d}, \mathrm{~J}=25.9 \mathrm{~Hz}), 110.50(\mathrm{~d}, \mathrm{~J}=25.3 \mathrm{~Hz}), 85.98(\mathrm{C} 2), 52.27(\mathrm{C} 5) ; \mathrm{MS}:(\mathrm{m} / \mathrm{z})$ $290\left([\mathrm{M}+\mathrm{H}]^{+}\right), \mathrm{C}_{15} \mathrm{H}_{13} \mathrm{O}_{2} \mathrm{NSF}(290.07)$.

Sulfoxide-3-(4-fluorophenyl)-2-phenyl-1,3-thiazolidin-4-one (2g). Yield (99\%); $\mathrm{R}_{\mathrm{f}}=0.230 ;$ m.p. 188-190 ${ }^{\circ} \mathrm{C}$; IR: $\mathrm{cm}^{-1} 1705.0(\mathrm{C}=\mathrm{O}), 1046.8(\mathrm{~S}=\mathrm{O}) ;{ }^{1} \mathrm{H}$ NMR $\left(\mathrm{CDCl}_{3}\right): 7.51-6.96(9 \mathrm{H}$, aromatics $)$. Major isomer $(96 \%), 5.89(1 \mathrm{H}, \mathrm{s}$, $\mathrm{C} 2), 3.90(1 \mathrm{H}, \mathrm{d}, \mathrm{C} 5, \mathrm{~J}=16.9 \mathrm{~Hz}), 3.58(1 \mathrm{H}, \mathrm{dd}, \mathrm{C} 5 \mathrm{~J}=1.1 \mathrm{~Hz}$ and $\mathrm{J}=17.0 \mathrm{~Hz})$. Minor isomer $(4 \%), 6.01(1 \mathrm{H}, \mathrm{s}, \mathrm{C} 2)$, $3.98(1 \mathrm{H}, \mathrm{d}, \mathrm{C} 5, \mathrm{~J}=16.5 \mathrm{~Hz}), 3.81(1 \mathrm{H}, \mathrm{d}, \mathrm{C} 5, \mathrm{~J}=16.7 \mathrm{~Hz}) ;{ }^{13} \mathrm{C}$ NMR: $168.55(\mathrm{C} 4), 161.28(\mathrm{~d}, \mathrm{~J}=231.2), 134.05(\mathrm{~d}, \mathrm{~J}$ $=4.3 \mathrm{~Hz}), 130.64,130.34,130.17,129.14(\mathrm{~d}, \mathrm{~J}=4.0 \mathrm{~Hz}), 126.76(\mathrm{~d}, \mathrm{~J}=8.5), 126.44,125.79(\mathrm{~J}=9.6 \mathrm{~Hz}), 116.45(\mathrm{~d}, \mathrm{~J}$ $=25.5 \mathrm{~Hz}), 86.82(\mathrm{C} 2), 52.27(\mathrm{C} 5) ; \mathrm{MS}:(\mathrm{m} / \mathrm{z}) 290\left([\mathrm{M}+\mathrm{H}]^{+}\right), \mathrm{C}_{15} \mathrm{H}_{13} \mathrm{O}_{2} \mathrm{NSF}(290.07)$.

Sulfoxide-3-(3-bromophenyl)-2-phenyl-1,3-thiazolidin-4-one (2h). Yield (75\%); $\mathrm{R}_{\mathrm{f}}=0.112$; m.p. 167-169 ${ }^{\circ} \mathrm{C}$; IR: $\mathrm{cm}^{-1} 1683.9(\mathrm{C}=\mathrm{O}), 1052.8(\mathrm{~S}=\mathrm{O}) ;{ }^{1} \mathrm{H}$ NMR $\left(\mathrm{CDCl}_{3}\right): 7.64-7.04(9 \mathrm{H}$, aromatics $)$. Major isomer $(94 \%), 5.92(1 \mathrm{H}, \mathrm{s}$, $\mathrm{C} 2), 3.80(1 \mathrm{H}, \mathrm{d}, \mathrm{C} 5, \mathrm{~J}=16.9 \mathrm{~Hz}), 3.49(1 \mathrm{H}, \mathrm{dd}, \mathrm{C} 5 \mathrm{~J}=1.2 \mathrm{~Hz}$ and $\mathrm{J}=17.0 \mathrm{~Hz})$. Minor isomer $(6 \%), 6.00(1 \mathrm{H}, \mathrm{s}, \mathrm{C} 2)$, $3.92(1 \mathrm{H}, \mathrm{d}, \mathrm{C} 5, \mathrm{~J}=16.7 \mathrm{~Hz}), 3.70(1 \mathrm{H}, \mathrm{d}, \mathrm{C} 5 \mathrm{~J}=16.5 \mathrm{~Hz}) ;{ }^{13} \mathrm{C}$ NMR: 168.47 (C4), 139.29, 130.53, 130.18, 130.02, 129.91, 128.96, 128.93, 126.23, 122.79, 121.52, 85.91 (C2), 52.17 (C5); MS: (m/z) $350\left([\mathrm{M}+\mathrm{H}]^{+}\right), \mathrm{C}_{15} \mathrm{H}_{13} \mathrm{O}_{2} \mathrm{NSBr}$ (349.99).

Sulfoxide-3-(4-bromophenyl)-2-phenyl-1,3-thiazolidin-4-one (2i). Yield (99\%); $\mathrm{R}_{\mathrm{f}}=0.134 ; \mathrm{m} . \mathrm{p} .190-191{ }^{\circ} \mathrm{C}$; IR: $\mathrm{cm}^{-1} 1715.0(\mathrm{C}=\mathrm{O}), 1048.5(\mathrm{~S}=\mathrm{O}) ;{ }^{1} \mathrm{H}$ NMR $\left(\mathrm{CDCl}_{3}\right): 7.46-7.09(9 \mathrm{H}$, aromatics $)$. Major isomer $(89 \%), 5.91(1 \mathrm{H}, \mathrm{s}$, $\mathrm{C} 2), 3.84(1 \mathrm{H}, \mathrm{d}, \mathrm{C} 5, \mathrm{~J}=17.3 \mathrm{~Hz}), 3.54(1 \mathrm{H}, \mathrm{dd}, \mathrm{C} 5 \mathrm{~J}=1.3 \mathrm{~Hz}$ and $\mathrm{J}=17.0 \mathrm{~Hz})$. Minor isomer $(11 \%), 5.95(1 \mathrm{H}, \mathrm{s}, \mathrm{C} 2)$, $3.95(1 \mathrm{H}, \mathrm{d}, \mathrm{C} 5, \mathrm{~J}=16.7 \mathrm{~Hz}), 3.77(1 \mathrm{H}, \mathrm{d}, \mathrm{C} 5 \mathrm{~J}=16.7 \mathrm{~Hz}) ;{ }^{13} \mathrm{C}$ NMR: $168.42(\mathrm{C} 4), 137.18,132.54,132.23,130.43$, 130.39, 130.34, 130.17, 129.17, 129.02, 126.34, 124.81, 120.37, 86.23 (C2), 53.79 (C5), 52.17 (C5); MS: (m/z) 350 $\left([\mathrm{M}+\mathrm{H}]^{+}\right), \mathrm{C}_{15} \mathrm{H}_{13} \mathrm{O}_{2} \mathrm{NSBr}(349.99)$.

Sulfoxide-3-(3-methylphenyl)-2-phenyl-1,3-thiazolidin-4-one (2j). Yield (97\%); $\mathrm{R}_{\mathrm{f}}=0.143 ; \mathrm{m} . \mathrm{p} .161-162{ }^{\circ} \mathrm{C}$; IR: $\mathrm{cm}^{-1}$ 1678.2 $(\mathrm{C}=\mathrm{O}), 1048.4(\mathrm{~S}=\mathrm{O}) ;{ }^{1} \mathrm{H}$ NMR $\left(\mathrm{CDCl}_{3}\right): 7.42-6.98(9 \mathrm{H}$, aromatics). Major isomer $(96 \%), 5.95(1 \mathrm{H}, \mathrm{s}$, $\mathrm{C} 2), 3.86(1 \mathrm{H}, \mathrm{d}, \mathrm{C} 5, \mathrm{~J}=17.0 \mathrm{~Hz}), 3.57(1 \mathrm{H}, \mathrm{dd}, \mathrm{C} 5 \mathrm{~J}=1.1 \mathrm{~Hz}$ and $\mathrm{J}=17.0 \mathrm{~Hz})$. Minor isomer $(4 \%), 6.06(1 \mathrm{H}, \mathrm{s}, \mathrm{C} 2)$, $3.95(1 \mathrm{H}, \mathrm{d}, \mathrm{C} 5, \mathrm{~J}=16.7 \mathrm{~Hz}), 3.70(1 \mathrm{H}, \mathrm{d}, \mathrm{C} 5 \mathrm{~J}=16.5 \mathrm{~Hz}), 2.24(3 \mathrm{H}, \mathrm{s}) ;{ }^{13} \mathrm{C}$ NMR: $168.43(\mathrm{C} 4), 139.38,137.81$, 
130.67, 129.93, 129.82, 129.09, 128.71, 127.94, 126.35, 124.71, 124.44, 120.68, 120.45, 116.91, 86.46 (C2), 52.19 (C5), $21.41\left(\mathrm{CH}_{3}\right) ; \mathrm{MS}:(\mathrm{m} / \mathrm{z}) 286\left([\mathrm{M}+\mathrm{H}]^{+}\right), \mathrm{C}_{16} \mathrm{H}_{16} \mathrm{O}_{2} \mathrm{NS}(286.09)$.

Sulfoxide-3-(4-methylphenyl)-2-phenyl-1,3-thiazolidin-4-one (2k). Yield (97\%); $\mathrm{R}_{\mathrm{f}}=0.157$; m.p. 179-180 ${ }^{\circ} \mathrm{C}$; IR: $\mathrm{cm}^{-1} 1704.9(\mathrm{C}=\mathrm{O}), 1051.1(\mathrm{~S}=\mathrm{O}) ;{ }^{1} \mathrm{H}$ NMR $\left(\mathrm{CDCl}_{3}\right): 7.44-6.96(9 \mathrm{H}$, aromatics), $5.91(1 \mathrm{H}, \mathrm{s}, \mathrm{C} 2), 4.04(1 \mathrm{H}, \mathrm{d}, \mathrm{C} 5, \mathrm{~J}$ $=16.7 \mathrm{~Hz}), 3.99(1 \mathrm{H}, \mathrm{d}, \mathrm{C} 5 \mathrm{~J}=16.7 \mathrm{~Hz}), 2.29(3 \mathrm{H}, \mathrm{s}) ;{ }^{13} \mathrm{C}$ NMR: $168.34(\mathrm{C} 4), 137.02,135.32,130.80,129.93(\mathrm{br})$, 129.84, 126.41, 123.66, 86.57 (C2), 52.24 (C5), $21.01\left(\mathrm{CH}_{3}\right) ; \mathrm{MS}:(\mathrm{m} / \mathrm{z}) 286\left([\mathrm{M}+\mathrm{H}]^{+}\right), \mathrm{C}_{16} \mathrm{H}_{16} \mathrm{O}_{2} \mathrm{NS}(286.09)$.

Sulfoxide-3-(3-methoxyphenyl)-2-phenyl-1,3-thiazolidin-4-one (2l). Yield (99\%); $\mathrm{R}_{\mathrm{f}}=0.056 ; \mathrm{m} . \mathrm{p} . \mathrm{d} 219{ }^{\circ} \mathrm{C} ; \mathrm{IR}: \mathrm{cm}^{-1}$ 1693.2 $(\mathrm{C}=\mathrm{O}), 1027.7(\mathrm{~S}=\mathrm{O}) ;{ }^{1} \mathrm{H}$ NMR $\left(\mathrm{CDCl}_{3}\right)$ : $7.55-6.66(9 \mathrm{H}$, aromatics). Major isomer $(91 \%), 5.46(1 \mathrm{H}, \mathrm{s}, \mathrm{C} 2)$, $3.62(2 \mathrm{H}, \mathrm{s}, \mathrm{C} 5)$. Minor isomer (9\%), $5.64(1 \mathrm{H}, \mathrm{s}, \mathrm{C} 2), 3.80(1 \mathrm{H}, \mathrm{d}, \mathrm{C} 5, \mathrm{~J}=1.7 \mathrm{~Hz}), 3.78(3 \mathrm{H}$ and minor isomer's C5, s); ${ }^{13}$ C NMR: 165.96 (C4), 138.17, 133.46, 131.56, 131.18, 130.51, 130.26, 129.59, 129.34, 129.17, 129.01, 122.40, 112.63, 110.10, $71.45(\mathrm{C} 2), 55.84\left(\mathrm{OCH}_{3}\right), 52.19(\mathrm{C} 5)$; MS: (m/z) $302\left([\mathrm{M}+\mathrm{H}]^{+}\right), \mathrm{C}_{16} \mathrm{H}_{16} \mathrm{O}_{3} \mathrm{NS}(302.09)$.

Sulfoxide-3-(4-methoxyphenyl)-2-phenyl-1,3-thiazolidin-4-one (2m). Yield (99\%); $\mathrm{R}_{\mathrm{f}}=0.101$; m.p. 188-189 ${ }^{\circ} \mathrm{C}$; IR: $\mathrm{cm}^{-1}$ 1697.7 $(\mathrm{C}=\mathrm{O}), 1049.4,1027.2(\mathrm{~S}=\mathrm{O}) ;{ }^{1} \mathrm{H}$ NMR $\left(\mathrm{CDCl}_{3}\right): 7.49-6.84(9 \mathrm{H}$, aromatics). Major isomer $(93 \%), 5.86$ $(1 \mathrm{H}, \mathrm{s}, \mathrm{C} 2), 3.91(1 \mathrm{H}, \mathrm{d}, \mathrm{C} 5, \mathrm{~J}=17.2 \mathrm{~Hz}), 3.56(1 \mathrm{H}, \mathrm{dd}, \mathrm{J}=0.9 \mathrm{~Hz}$ and $\mathrm{J}=17.2 \mathrm{~Hz})$. Minor isomer $(7 \%), 5.99(1 \mathrm{H}, \mathrm{s}$, C2), $3.97(1 \mathrm{H}, \mathrm{d}, \mathrm{C} 5, \mathrm{~J}=16.3 \mathrm{~Hz}), 3.76(3 \mathrm{H}$ and minor isomer's $\mathrm{C} 5, \mathrm{~s}) ;{ }^{13} \mathrm{C}$ NMR: $168.49(\mathrm{C} 4), 158.65,130.97,130.66$, 130.16, 130.04, 129.13, 129.03, 126.54, 126.38, 125.80, 114.68, 114.49, $87.16(\mathrm{C} 2), 55.63\left(\mathrm{OCH}_{3}\right), 52.31(\mathrm{C} 5)$; MS: $(\mathrm{m} / \mathrm{z}) 302\left([\mathrm{M}+\mathrm{H}]^{+}\right), \mathrm{C}_{16} \mathrm{H}_{16} \mathrm{O}_{3} \mathrm{NS}(302.09)$.

Sulfoxide-3-(3-nitrophenyl)-2-phenyl-1,3-thiazolidin-4-one (2n). Yield (99\%); $R_{\mathrm{f}}=0.089 ;$ m.p. 205-207 ${ }^{\circ} \mathrm{C}$; IR: $\mathrm{cm}^{-1}$ $1702.9(\mathrm{C}=\mathrm{O}), 1062.4(\mathrm{br}, \mathrm{S}=\mathrm{O}) ;{ }^{1} \mathrm{H}$ NMR $\left(\mathrm{CDCl}_{3}\right): 8.28-7.19(9 \mathrm{H}$, aromatics). Major isomer $(90 \%), 5.97(1 \mathrm{H}, \mathrm{s}, \mathrm{C} 2)$, $3.86(1 \mathrm{H}, \mathrm{d}, \mathrm{C} 5, \mathrm{~J}=17.0 \mathrm{~Hz}), 3.58(1 \mathrm{H}, \mathrm{dd}, \mathrm{J}=1.2 \mathrm{~Hz}$ and $\mathrm{J}=17.1 \mathrm{~Hz})$. Minor isomer $(10 \%), 6.05(1 \mathrm{H}, \mathrm{s}, \mathrm{C} 2), 3.96$ $(1 \mathrm{H}, \mathrm{d}, \mathrm{C} 5, \mathrm{~J}=17.1 \mathrm{~Hz}), 3.84(1 \mathrm{H}, \mathrm{d}, \mathrm{J}=17.1 \mathrm{~Hz}) ;{ }^{13} \mathrm{C}$ NMR: $168.73(\mathrm{C} 4), 148.83,139.41,130.74,130.49,130.40$, $129.83,129.48,129.17,128.49,126.26,121.63,121.52,117.79,86.03(\mathrm{C} 2), 52.38(\mathrm{C} 5) ; \mathrm{MS}:(\mathrm{m} / \mathrm{z}) 317\left([\mathrm{M}+\mathrm{H}]^{+}\right)$, $\mathrm{C}_{15} \mathrm{H}_{13} \mathrm{O}_{4} \mathrm{~N}_{2} \mathrm{~S}$ (317.06).

Sulfoxide-3-(4-nitrophenyl)-2-phenyl-1,3-thiazolidin-4-one (2o). Yield (44\%); $\mathrm{R}_{\mathrm{f}}=0.075 ; \mathrm{m} . \mathrm{p} . \mathrm{d} 213{ }^{\circ} \mathrm{C}$; IR: $\mathrm{cm}^{-1}$ 1706.2 (C=O), $1051.4(\mathrm{br}, \mathrm{S}=\mathrm{O}) ;{ }^{1} \mathrm{H}$ NMR $\left(\mathrm{CDCl}_{3}\right): 8.22-7.35(9 \mathrm{H}$, aromatics), $6.08(1 \mathrm{H}, \mathrm{s}, \mathrm{C} 2), 3.90(1 \mathrm{H}, \mathrm{d}, \mathrm{C} 5, \mathrm{~J}=$ $17.4 \mathrm{~Hz}), 3.65(1 \mathrm{H}, \mathrm{dd}, \mathrm{J}=1.2 \mathrm{~Hz}$ and $\mathrm{J}=17.3 \mathrm{~Hz}) ;{ }^{13} \mathrm{C}$ NMR: $168.83(\mathrm{C} 4), 145.16,143.92,130.71,130.47,129.68$, 126.16, 125.00, 122.07, 85.64 (C2), 52.41 (C5); MS: (m/z) $317\left([\mathrm{M}+\mathrm{H}]^{+}\right), \mathrm{C}_{15} \mathrm{H}_{13} \mathrm{O}_{4} \mathrm{~N}_{2} \mathrm{~S}$ (317.06).

4.3 General Procedure for the Synthesis of Thiazolidin-4-One Sulfones via $\mathrm{KMnO}_{4}$

Thiazolidin-4-one $(0.553 \mathrm{mmol})$ was dissolved in glacial acetic acid $(2.4 \mathrm{~mL})$, to which an aqueous solution of $\mathrm{KMnO}_{4}$ $(175 \mathrm{mg}, 1.11 \mathrm{mmol}$, in $3.0 \mathrm{~mL}$ water) was added dropwise at room temperature with vigorous stirring, and stirred an additional $5 \mathrm{~m}$. Solid sodium bisulfite $\left(\mathrm{NaHSO}_{3} / \mathrm{Na}_{2} \mathrm{~S}_{2} \mathrm{O}_{5}\right)$ was then added until the solution remained colorless; $3.0 \mathrm{~mL}$ of water was then added to the mixture and stirred for $10 \mathrm{~m}$. Most crude products were isolated as powders by filtration and water rinses; products were purified by recrystallization in $\mathrm{CH}_{3} \mathrm{OH}$. Products $\mathbf{3 a}, \mathbf{3 c}$, and $\mathbf{3 m}$ were not isolated as powders, but rather by extraction of the reaction mixture with toluene $(3 \times 10 \mathrm{~mL})$. The combined toluene layers were dried with $\mathrm{Na}_{2} \mathrm{SO}_{4}$, and toluene was removed in vacuo followed by chromatography.

Sulfone-2-phenyl-2-(3-trifluoromethylphenyl)-1,3-thiazolidin-4-one (3a). Yield: (78\%); $\mathrm{R}_{\mathrm{f}}=0.219 ;$ m.p. 106-110 ${ }^{\circ} \mathrm{C}$; IR cm ${ }^{-1} 1702.6(\mathrm{C}=\mathrm{O}), 1126.2,1114.1(\mathrm{~S}=\mathrm{O}) ;{ }^{1} \mathrm{H}$ NMR $\left(\mathrm{CDCl}_{3}\right), 7.71-7.33(9 \mathrm{H}$, aromatics $), 6.13(1 \mathrm{H}, \mathrm{s}, \mathrm{C} 2), 4.16$ $(1 \mathrm{H}, \mathrm{d}, \mathrm{C} 5, \mathrm{~J}=17.1 \mathrm{~Hz}), 4.12(1 \mathrm{H}, \mathrm{d}, \mathrm{C} 5 \mathrm{~J}=17.1 \mathrm{~Hz}) ;{ }^{13} \mathrm{C}$ NMR: $162.72(\mathrm{C} 4), 131.84(\mathrm{q}, \mathrm{J}=33.1), 131.05,130.23$, 130.08, 129.7, 128.61, 128.22, 123.43 (q, J = 272.4 Hz), 124.51 (br q, J = 3.2 Hz), 121.93 (br q, J = 3.9 Hz), $83.22(\mathrm{C} 2)$, 50.67 (C5); MS: (m/z) $356\left([\mathrm{M}+\mathrm{H}]^{+}\right), \mathrm{C}_{16} \mathrm{H}_{13} \mathrm{O}_{3} \mathrm{NSF}_{3}(356.06)$.

Sulfone-2-phenyl-2-(4-trifluoromethylphenyl)-1,3-thiazolidin-4-one (3b). Yield: (81\%); m.p. 186-187 ${ }^{\circ} \mathrm{C}$; IR cm ${ }^{-1}$ $1697.8(\mathrm{C}=\mathrm{O}), 1123.1(\mathrm{~S}=\mathrm{O}) ;{ }^{1} \mathrm{H}$ NMR $\left(\mathrm{CDCl}_{3}\right), 7.60-7.36(9 \mathrm{H}$, aromatics $), 6.01(1 \mathrm{H}, \mathrm{s}, \mathrm{C} 2), 4.09(1 \mathrm{H}, \mathrm{d}, \mathrm{C} 5, \mathrm{~J}=$ $16.6 \mathrm{~Hz}), 4.04(1 \mathrm{H}, \mathrm{d}, \mathrm{C} 5, \mathrm{~J}=16.6 \mathrm{~Hz}) ;{ }^{13} \mathrm{C}$ NMR: $162.62(\mathrm{C} 4), 139.33,129.88,129.78,129.61$ (q, J = 32.4 Hz), 128.43, 127.90, $126.69(\mathrm{q}, \mathrm{J}=4.2 \mathrm{~Hz}), 124.76,123.64(\mathrm{q}, \mathrm{J}=272.1 \mathrm{~Hz}), 83.23(\mathrm{C} 2), 50.69(\mathrm{C} 5) ; \mathrm{MS}:(\mathrm{m} / \mathrm{z}) 356\left([\mathrm{M}+\mathrm{H}]^{+}\right)$, $\mathrm{C}_{16} \mathrm{H}_{13} \mathrm{O}_{3} \mathrm{NSF}_{3}$ (356.06).

Sulfone-2,3-diphenyl-1,3-thiazolidin-4-one (3c). Yield (76\%); $\mathrm{R}_{\mathrm{f}}=0.366$; m.p. 178-180 ${ }^{\circ} \mathrm{C}$ : IR: $\mathrm{cm}^{-1} 1693.1(\mathrm{C}=\mathrm{O})$, 1141.6, $1125.2(\mathrm{~S}=\mathrm{O}) ;{ }^{1} \mathrm{H}$ NMR ( $\left.\mathrm{CDCl}_{3}\right): 7.47-7.18(10 \mathrm{H}$, aromatics), $5.99(1 \mathrm{H}, \mathrm{s}, \mathrm{C} 2), 3.88(1 \mathrm{H}, \mathrm{d}, \mathrm{C} 5, \mathrm{~J}=16.9 \mathrm{~Hz})$, $3.56(1 \mathrm{H}, \mathrm{dd}, \mathrm{C} 5 \mathrm{~J}=1.2 \mathrm{~Hz}$ and $\mathrm{J}=17.1 \mathrm{~Hz}) ;{ }^{13} \mathrm{C}$ NMR: $162.58(\mathrm{C} 4), 136.25,130.87,129.62,129.58,129.01,128.05$, 125.13, $83.92(\mathrm{C} 2), 50.70(\mathrm{C} 5)$; MS: (m/z) $288\left([\mathrm{M}+\mathrm{H}]^{+}\right), \mathrm{C}_{15} \mathrm{H}_{14} \mathrm{O}_{3} \mathrm{NS}(288.07)$.

Sulfone-3-(3-chlorophenyl)-2-phenyl-1,3-thiazolidin-4-one (3d). Yield (82\%); m.p. 183-184 ${ }^{\circ} \mathrm{C}$; IR: $\mathrm{cm}^{-1} 1694.8$ $(\mathrm{C}=\mathrm{O}), 1130.4(\mathrm{~S}=\mathrm{O}) ;{ }^{1} \mathrm{H}$ NMR $\left(\mathrm{CDCl}_{3}\right): 7.47-7.13(9 \mathrm{H}$, aromatics $), 5.92(1 \mathrm{H}, \mathrm{s}, \mathrm{C} 2), 4.04(1 \mathrm{H}, \mathrm{d}, \mathrm{C} 5, \mathrm{~J}=16.7 \mathrm{~Hz})$, $4.00(1 \mathrm{H}, \mathrm{d}, \mathrm{C} 5 \mathrm{~J}=16.7 \mathrm{~Hz}) ;{ }^{13} \mathrm{C}$ NMR: $162.51(\mathrm{C} 4), 137.31,135.19,131.11,130.51,129.82,128.62,128.24,127.93$, 125.34, 122.98, $83.62(\mathrm{C} 2), 50.64(\mathrm{C} 5)$; MS: (m/z) $322\left([\mathrm{M}+\mathrm{H}]^{+}\right), \mathrm{C}_{15} \mathrm{H}_{13} \mathrm{O}_{3} \mathrm{NSCl}(322.03)$. 
Sulfone-3-(4-chlorophenyl)-2-phenyl-1,3-thiazolidin-4-one (3e). Yield (76\%); m.p. 176-178 ${ }^{\circ} \mathrm{C}$; IR: $\mathrm{cm}^{-1} 1702.9$ $(\mathrm{C}=\mathrm{O}), 1129.3,1089.6(\mathrm{~S}=\mathrm{O}) ;{ }^{1} \mathrm{H}$ NMR $\left(\mathrm{CDCl}_{3}\right): 7.41-7.16(9 \mathrm{H}$, aromatics $), 5.87(1 \mathrm{H}, \mathrm{s}, \mathrm{C} 2), 4.00(1 \mathrm{H}, \mathrm{d}, \mathrm{C} 5, \mathrm{~J}=$ $16.8 \mathrm{~Hz}), 3.96(1 \mathrm{H}, \mathrm{d}, \mathrm{C} 5 \mathrm{~J}=16.3 \mathrm{~Hz}) ;{ }^{13} \mathrm{C}$ NMR: $162.54(\mathrm{C} 4), 134.68,133.87,131.10,129.78,129.76,128.56,128.06$, 126.33, $83.66(\mathrm{C} 2), 50.71(\mathrm{C} 5) ; \mathrm{MS}:(\mathrm{m} / \mathrm{z}) 322\left([\mathrm{M}+\mathrm{H}]^{+}\right), \mathrm{C}_{15} \mathrm{H}_{13} \mathrm{O}_{3} \mathrm{NSCl}(322.03)$.

Sulfone-3-(3-fluorophenyl)-2-phenyl-1,3-thiazolidin-4-one (3f). Yield (77\%); m.p. 171-172 ${ }^{\circ} \mathrm{C}$; IR: $\mathrm{cm}^{-1} 1707.3$ $(\mathrm{C}=\mathrm{O}), 1058.8(\mathrm{~S}=\mathrm{O}) ;{ }^{1} \mathrm{H}$ NMR $\left(\mathrm{CDCl}_{3}\right): 7.42-6.88(9 \mathrm{H}$, aromatics $), 5.90(1 \mathrm{H}, \mathrm{s}, \mathrm{C} 2), 3.99(2 \mathrm{H}$, overlapping d, C5, J $=17.5 \mathrm{~Hz}) ;{ }^{13} \mathrm{C}$ NMR: $162.81(\mathrm{~d}, \mathrm{~J}=246.3), 162.53(\mathrm{C} 4), 137.61(\mathrm{~d}, \mathrm{~J}=9.7 \mathrm{~Hz}), 131.06,130.74(\mathrm{~d}, \mathrm{~J}=9.6 \mathrm{~Hz}), 129.80$, 128.69, 127.90, $120.23(\mathrm{~d}, \mathrm{~J}=2.4), 112.61(\mathrm{~d}, \mathrm{~J}=25.2 \mathrm{~Hz}), 83.54(\mathrm{C} 2), 50.70(\mathrm{C} 5) ; \mathrm{MS}:(\mathrm{m} / \mathrm{z}) 306\left([\mathrm{M}+\mathrm{H}]^{+}\right)$, $\mathrm{C}_{15} \mathrm{H}_{13} \mathrm{O}_{3} \mathrm{NSF}$ (306.06).

Sulfone-3-(4-fluorophenyl)-2-phenyl-1,3-thiazolidin-4-one (3g). Yield (56\%); m.p. 141-142 ${ }^{\circ} \mathrm{C}$; IR: $\mathrm{cm}^{-1} 1699.0$ $(\mathrm{C}=\mathrm{O}), 1142.0,1122.5(\mathrm{~S}=\mathrm{O}) ;{ }^{1} \mathrm{H}$ NMR $\left(\mathrm{CDCl}_{3}\right): 7.45-6.96(9 \mathrm{H}$, aromatics $), 5.91(1 \mathrm{H}, \mathrm{s}, \mathrm{C} 2), 4.04(1 \mathrm{H}, \mathrm{d}, \mathrm{C} 5, \mathrm{~J}=$ $16.7 \mathrm{~Hz}), 3.99(1 \mathrm{H}, \mathrm{d}, \mathrm{C} 5 \mathrm{~J}=16.8 \mathrm{~Hz}) ;{ }^{13} \mathrm{C}$ NMR: $162.83(\mathrm{C} 4), 161.52(\mathrm{~d}, \mathrm{~J}=236.1), 132.05(\mathrm{~d}, \mathrm{~J}=3.5 \mathrm{~Hz}), 131.00$, 129.67, 128.62, 128.16, $127.25(\mathrm{~d}, \mathrm{~J}=9.4 \mathrm{~Hz}), 116.57(\mathrm{~d}, \mathrm{~J}=22.5 \mathrm{~Hz}), 83.93(\mathrm{C} 2), 50.64$ (C5); MS: (m/z) 306 $\left([\mathrm{M}+\mathrm{H}]^{+}\right), \mathrm{C}_{15} \mathrm{H}_{13} \mathrm{O}_{3} \mathrm{NSF}(306.06)$.

Sulfone-3-(3-bromophenyl)-2-phenyl-1,3-thiazolidin-4-one (3h). Yield (77\%); m.p. 190-191 ${ }^{\circ} \mathrm{C}$; IR: $\mathrm{cm}^{-1} 1693.8$

$(\mathrm{C}=\mathrm{O}), 1128.1(\mathrm{~S}=\mathrm{O}) ;{ }^{1} \mathrm{H}$ NMR $\left(\mathrm{CDCl}_{3}\right): 7.55-7.17(9 \mathrm{H}$, aromatics $), 5.94(1 \mathrm{H}, \mathrm{s}, \mathrm{C} 2), 4.06(1 \mathrm{H}, \mathrm{d}, \mathrm{C} 5, \mathrm{~J}=16.7 \mathrm{~Hz})$, $4.02(1 \mathrm{H}, \mathrm{d}, \mathrm{C} 5 \mathrm{~J}=16.7 \mathrm{~Hz}) ;{ }^{13} \mathrm{C}$ NMR: $162.51(\mathrm{C} 4), 137.43,131.19,131.12,130.77,129.84,128.62,128.21,127.94$, 123.50, 123.00, $83.66(\mathrm{C} 2), 50.64(\mathrm{C} 5)$; MS: (m/z) $366\left([\mathrm{M}+\mathrm{H}]^{+}\right), \mathrm{C}_{15} \mathrm{H}_{13} \mathrm{O}_{3} \mathrm{NSBr}(365.98)$.

Sulfone-3-(4-bromophenyl)-2-phenyl-1,3-thiazolidin-4-one (3i). Yield (76\%); m.p. 201-204 ${ }^{\circ} \mathrm{C}$; IR: $\mathrm{cm}^{-1} 1698.8$ $(\mathrm{C}=\mathrm{O}), 1128.6(\mathrm{~S}=\mathrm{O}) ;{ }^{1} \mathrm{H}$ NMR $\left(\mathrm{CDCl}_{3}\right): 7.49-7.16(9 \mathrm{H}$, aromatics $), 5.93(1 \mathrm{H}, \mathrm{s}, \mathrm{C} 2), 4.07(1 \mathrm{H}, \mathrm{d}, \mathrm{C} 5, \mathrm{~J}=16.7 \mathrm{~Hz})$, $4.02(1 \mathrm{H}, \mathrm{d}, \mathrm{C} 5 \mathrm{~J}=16.8 \mathrm{~Hz}) ;{ }^{13} \mathrm{C}$ NMR: $162.46(\mathrm{C} 4), 135.24,132.74,131.12,129.81,128.57,128.03,126.56,121.63$, 83.62 (C2), 50.73 (C5); MS: (m/z) $366\left([\mathrm{M}+\mathrm{H}]^{+}\right), \mathrm{C}_{15} \mathrm{H}_{13} \mathrm{O}_{3} \mathrm{NSBr}(365.98)$.

Sulfone-3-(3-methylphenyl)-2-phenyl-1,3-thiazolidin-4-one (3j). Yield (65\%); m.p. 173-175 ${ }^{\circ} \mathrm{C}$; IR: $\mathrm{cm}^{-1} 1687.2$ $(\mathrm{C}=\mathrm{O}), 1138.5,1124.0(\mathrm{~S}=\mathrm{O}) ;{ }^{1} \mathrm{H}$ NMR $\left(\mathrm{CDCl}_{3}\right): 7.47-7.03(9 \mathrm{H}$, aromatics), $5.95(1 \mathrm{H}, \mathrm{s}, \mathrm{C} 2), 4.05(1 \mathrm{H}, \mathrm{d}, \mathrm{C} 5, \mathrm{~J}=$ $16.5 \mathrm{~Hz}), 4.00(1 \mathrm{H}, \mathrm{d}, \mathrm{C} 5, \mathrm{~J}=16.5 \mathrm{~Hz}), 2.30(3 \mathrm{H}, \mathrm{s}) ;{ }^{13} \mathrm{C}$ NMR: $162.60(\mathrm{C} 4), 139.75,136.16,130.84,129.62,129.37$, 129.20, 129.04, 128.00, 125.97, 122.22, $84.14(\mathrm{C} 2), 50.68(\mathrm{C} 5), 21.55\left(\mathrm{CH}_{3}\right) ; \mathrm{MS}:(\mathrm{m} / \mathrm{z}) 302\left([\mathrm{M}+\mathrm{H}]^{+}\right), \mathrm{C}_{16} \mathrm{H}_{16} \mathrm{O}_{3} \mathrm{NS}$ (302.09).

Sulfone-3-(4-methylphenyl)-2-phenyl-1,3-thiazolidin-4-one (3k). Yield (78\%); m.p. 104-105 ${ }^{\circ} \mathrm{C}$; IR: $\mathrm{cm}^{-1} 1701.9$ $(\mathrm{C}=\mathrm{O}), 1128.0(\mathrm{~S}=\mathrm{O}) ;{ }^{1} \mathrm{H}$ NMR $\left(\mathrm{CDCl}_{3}\right): 7.45-7.11(9 \mathrm{H}$, aromatics $), 5.95(1 \mathrm{H}, \mathrm{s}, \mathrm{C} 2), 4.05(1 \mathrm{H}, \mathrm{d}, \mathrm{C} 5, \mathrm{~J}=16.7 \mathrm{~Hz})$, $4.00(1 \mathrm{H}, \mathrm{d}, \mathrm{C} 5 \mathrm{~J}=16.6 \mathrm{~Hz}), 2.29(3 \mathrm{H}, \mathrm{s}) ;{ }^{13} \mathrm{C}$ NMR: $162.58(\mathrm{C} 4), 138.18,133.55,130.80,130.17,129.56,129.05$, 128.12, 125.12, $84.05(\mathrm{C} 2), 50.71(\mathrm{C} 5), 21.20\left(\mathrm{CH}_{3}\right)$; MS: (m/z) $302\left([\mathrm{M}+\mathrm{H}]^{+}\right), \mathrm{C}_{16} \mathrm{H}_{16} \mathrm{O}_{3} \mathrm{NS}(302.09)$.

Sulfone-3-(3-methoxyphenyl)-2-phenyl-1,3-thiazolidin-4-one (3I). Yield (83\%); m.p. 214-217 ${ }^{\circ} \mathrm{C}$; IR: $\mathrm{cm}^{-1} 1689.3$ $(\mathrm{C}=\mathrm{O}), 1129.1,1107.4(\mathrm{~S}=\mathrm{O}) ;{ }^{1} \mathrm{H}$ NMR $\left(\mathrm{CDCl}_{3}\right): 7.46-6.82(9 \mathrm{H}$, aromatics $), 5.64(1 \mathrm{H}, \mathrm{s}, \mathrm{C} 2),, 4.16(1 \mathrm{H}, \mathrm{d}, \mathrm{C} 5, \mathrm{~J}=$ $12.7 \mathrm{~Hz}), 3.97(1 \mathrm{H}, \mathrm{dd}, \mathrm{C} 5, \mathrm{~J}=1.7 \mathrm{~Hz}$ and $\mathrm{J}=12.7 \mathrm{~Hz}), 3.82(3 \mathrm{H}, \mathrm{s}) ;{ }^{13} \mathrm{C}$ NMR: $161.07(\mathrm{C} 4), 137.27,131.58,131.51$, 129.81, 129.31, 128.54, 120.38, 114.29, 109.87, 69.07 (C2), 55.90; MS: (m/z) $318\left([\mathrm{M}+\mathrm{H}]^{+}\right), \mathrm{C}_{16} \mathrm{H}_{16} \mathrm{O}_{4} \mathrm{NS}(318.08)$.

Sulfone-3-(4-methoxyphenyl)-2-phenyl-1,3-thiazolidin-4-one (3m). Yield $(95 \%)$; $\mathrm{R}_{\mathrm{f}}=0.265$; m.p. 104-105 ${ }^{\circ} \mathrm{C}$; IR: $\mathrm{cm}^{-1} 1694.1(\mathrm{C}=\mathrm{O}), 1126.8(\mathrm{~S}=\mathrm{O}) ;{ }^{1} \mathrm{H}$ NMR $\left(\mathrm{CDCl}_{3}\right): 7.44-6.81(9 \mathrm{H}$, aromatics $), 5.95(1 \mathrm{H}, \mathrm{s}, \mathrm{C} 2), 4.04(1 \mathrm{H}, \mathrm{d}, \mathrm{C} 5, \mathrm{~J}$ $=17.1 \mathrm{~Hz}), 3.99(1 \mathrm{H}, \mathrm{d}, \mathrm{J}=16.4 \mathrm{~Hz}), 3.73(3 \mathrm{H}, \mathrm{s}) ;{ }^{13} \mathrm{C}$ NMR: $162.76(\mathrm{C} 4), 158.93,130.71,129.44,128.86,128.66$, 128.25, 126.85, 114.67, 84.06 (C2), $55.51\left(\mathrm{OCH}_{3}\right), 50.63(\mathrm{C} 5)$; MS: (m/z) $318\left([\mathrm{M}+\mathrm{H}]^{+}\right), \mathrm{C}_{16} \mathrm{H}_{16} \mathrm{O}_{4} \mathrm{NS}(318.08)$.

Sulfone-3-(3-nitrophenyl)-2-phenyl-1,3-thiazolidin-4-one (3n). Yield (80\%); m.p. 199-201 ${ }^{\circ} \mathrm{C}$; IR: $\mathrm{cm}^{-1} 1698.0$ $(\mathrm{C}=\mathrm{O}), 1132.0(\mathrm{~S}=\mathrm{O}) ;{ }^{1} \mathrm{H}$ NMR $\left(\mathrm{CDCl}_{3}\right): 8.21-7.37\left(9 \mathrm{H}\right.$, aromatics), $6.04(1 \mathrm{H}, \mathrm{s}, \mathrm{C} 2), 4.11(2 \mathrm{H}, \mathrm{s}) ;{ }^{13} \mathrm{C}$ NMR: 162.63 (C4), 148.71, 137.25, 131.41, 130.50, 130.45, 130.04, 128.04, 127.88, 122.51, 119.79, 83.20 (C2), 50.74 (C5); MS: $(\mathrm{m} / \mathrm{z}) 333\left([\mathrm{M}+\mathrm{H}]^{+}\right), \mathrm{C}_{15} \mathrm{H}_{13} \mathrm{O}_{5} \mathrm{~N}_{2} \mathrm{~S}(333.05)$.

Sulfone-3-(4-nitrophenyl)-2-phenyl-1,3-thiazolidin-4-one (3o). Yield (56\%); m.p. 204-206 ${ }^{\circ} \mathrm{C}$; IR: $\mathrm{cm}^{-1} 1709.4(\mathrm{C}=\mathrm{O})$, 1125.7, $1111.7(\mathrm{~S}=\mathrm{O}) ;{ }^{1} \mathrm{H}$ NMR $\left(\mathrm{CDCl}_{3}\right): 8.17-7.35\left(9 \mathrm{H}\right.$, aromatics), $6.07(1 \mathrm{H}, \mathrm{s}, \mathrm{C} 2), 4.10(2 \mathrm{H}, \mathrm{s}) ;{ }^{13} \mathrm{C}$ NMR: 162.66 (C4), 145.86, 141.72, 131.34, 129.97, 127.90, 127.86, 124.86, 124.56, $82.72(\mathrm{C} 2), 52.41$ (C5); MS: (m/z) $333\left([\mathrm{M}+\mathrm{H}]^{+}\right)$, $\mathrm{C}_{15} \mathrm{H}_{13} \mathrm{O}_{4} \mathrm{~N}_{2} \mathrm{~S}$ (333.05).

4.4 High Temperature Oxidation of Thiazolidin-4-Ones 1a-o Using Oxone ${ }^{\circledR}$

Thiazolidin-4-one (1a-o) $(0.103 \mathrm{mmol})$ was dissolved in refluxing $\mathrm{MeOH}(16 \mathrm{~mL})$, to which a solution of Oxone ${ }^{\circledR}$ $\left(1.251 \mathrm{~g}, 0.824 \mathrm{mmol}\right.$, in $\left.8 \mathrm{~mL} \mathrm{H}_{2} \mathrm{O}\right)$ was added dropwise with vigorous stirring. Then the reaction mixture was heated for $8 \mathrm{~h}$. Upon cooling, $40 \mathrm{~mL}$ of $\mathrm{H}_{2} \mathrm{O}$ was added and the solution was then extracted with $\mathrm{CH}_{2} \mathrm{Cl}_{2}(3 \times 25 \mathrm{~mL})$. The combined $\mathrm{CH}_{2} \mathrm{Cl}_{2}$ layers were dried with $\mathrm{Na}_{2} \mathrm{SO}_{4}$, and the $\mathrm{CH}_{2} \mathrm{Cl}_{2}$ was removed in vacuo. The resulting crude product 
mixture of sulfoxide and sulfone was purified using preparative chromatography plates (silica gel GF, 250 micron) purchased from Analtech (Rabel \& Sherma, 2017). The product mixture was dissolved sparingly in $\mathrm{CH}_{2} \mathrm{Cl}_{2}$ and deposited on the preparative chromatography plates using a streaking apparatus purchased from the Aldrich Chemical Company. No more than $150 \mathrm{mg}$ of product mixture was deposited on a plate. The plates were developed using 3:1 cyclohexane: ethyl acetate, after which the plates were air-dried. The location of the products' bands on the plate were identified by $R_{f}$ values and fluorescent indicators present in the silica gel. The sulfoxide and sulfone bands were separated by scraping the bands of silica from the plate. The products were then isolated by extracting the collected silica with boiling ethyl acetate followed by filtration and removal of the ethyl acetate in vacuo. The molar ratio of sulfoxide $\mathbf{2}$ to sulfone $\mathbf{3}$ was determined by the isolated yields of the two respective compounds based on the thiazolidin-4-one $\mathbf{1}$ as the limiting reagent.

\section{Acknowledgements}

We would like to thank Dr. Charles DeBrosse and the Chemistry Department of Temple University for allowing us to run the NMR spectra on their spectrometer.

\section{References}

Abhinit, M., Ghodke, M., \& Pratima, N. A. (2009). Exploring potential of 4-thiazolidinone: a brief review. Int. J. Pharmacy Pharm. Sci., 1, 47-64.

Brown, F. C. (1961). 4-Thiazolidinones. Chem. Rev., 61, 463-521. https://doi.org/10.1021/cr60213a002

Cannon, K. C., Gandla, D., Lauro, S., Silverberg, L. J., Tierney, J., \& Lagalante, A. (2015). Selective synthesis of ortho-substituted 3-cyclohexyl-2-phenyl-1,3-thiazolidin-4-one sulfoxides and sulfones by $S$-oxidation with Oxone ${ }^{\circledR}$, Intl. J. Chem., 7(2), 73-83. https://doi.org/10.5539/ijc.v7n2p73

Colombo, A., Fernandez, J. C., Fernandez-Forner, D., de la Figuera, N., Albericio, F., \& Forns, P. (2008). Stereomeric studies on the oxidation and alkylation of 4-thiazolidinones. Tet. Lett., 49, 1569-1572. https://doi.org/10.1016/j.tetlet.2008.01.038

Gududuru, V., Hurh, E., Dalton, J. T., \& Miller, D. D. (2004). Discovery of 2-arylthiazolidine-4-carboxylic acid amides as a new class of cytotoxic agents for prostate cancer. Bioorg. Med. Chem. Lett., 14, 5289-5293. https://doi.org/10.1016/j.bmcl.2004.08.029

Hamama, W. S., Ismail, M. A., Shaaban, S., \& Zoorob, H. H. (2008). Progress in the chemistry of 4-thioazolidinones. J. Het. Chem., 45, 939-956. https://doi.org/10.1002/jhet.5570450401

Hussain, H., Green, I. R., \& Ahmed, I. (2013). Journey describing applications of oxone in synthetic chemistry. Chem. Rev., 113, 3329-3371. https://doi.org/10.1021/cr3004373

Jain, A. K., Vaidya, A., Ravichandran, V., Kashaw, S. K., \& Agrawal, R. A. (2012). Recent developments and biological activities of thiazolidinone derivatives: a review. Bioorg. Med. Chem., 20, 3378-3395. https://doi.org/10.1016/j.bmc.2012.03.069

Kaushal, M., \& Kaur, A. (2016). A review on some 2,5-disubstituted [1,3,4] thiadiazole substituted thiazolidinone derivatives as a potent antimicrobial agents. World Journal of Pharmaceutical Research, 5(6), 1966-1977.

Kumar, D., Kumar, V., Mundlia, J., Pradhan, D., \& Malik, S. (2015). Thiazolidin-4-one Derivatives as Central Nervous System Potential Agents. Central Nervous System Agents in Medicinal Chemistry, 15(1), 23-27. https://doi.org/10.2174/1871524915666150220111509

Lee, J. B., Yergatian, S. Y., Crowther, B. C., \& Downie, I. M. (1990). Preparation of 4-thiazolidinone 1-oxides. Org. Prep. Proc. Int., 22, 544-546. https://doi.org/10.1080/00304949009356324

Madesclaire, M. (1986). Reduction of sulfoxides to thioethers. Tetrahedron, 42, 5459-5480. https://doi.org/10.1016/S0040-4020(01)88150-3

Omar, M. T., El-Kharmry, A. E., \& Sherif, F. A. (1981). Synthesis of 2,3-disubstituted-4-oxothiazolidine 1-oxides. $J$. Het. Chem., 18, 633-634. https://doi.org/10.1002/jhet.5570180340

Rabel, F., \& Sherma, J. (2017). Review of the state of the art of preparative thin-layer chromatography. J. Liquid Chromatography and Related Technologies, 40(4), 165-176. https://doi.org/10.1080/10826076.2017.1294081

Rozwadowska, M. D., \& Sulima, A. (2003). Studies of some hydrogenated thiazolo[2,3-a]isoquinoline $S$-oxides. Tetrahedron, 59, 1173-1179. https://doi.org/10.1016/S0040-4020(03)00023-1

Rozwadowska, M. D., Sulima, A., \& Gzellla, A. (2002). Synthesis, crystal structure and oxidation of (R)-(+)-8,9-dimethoxy-6,10b-dihydro-5H-thiazolo[2,3-a]isoquinolin-3-one. $\quad$ Tet. Asymm., 13, 2329-2333. 
https://doi.org/10.1016/S0957-4166(02)00633-X

Singh, S. P., Parmar, S. S., Raman, R., Virgil I., \& Stenberg, V. I. (1981). Chemistry and biological activity of thiazolidinones. Chem. Rev., 81, 175-203. https://doi.org/10.1021/cr00042a003

Smith, R. L., Lee, T. J., \& Cragoe, E. J. (1977). Certain thiazolidine compounds. U.S. Patent 4059587.

Surrey, A. R. (1948). The preparation of 2,3-disubstituted-4-thiazolidones. II. 3-Alkyl (and aralkyl) 2-aryl derivatives. $J$. Am. Chem. Soc., 70, 4262-4263. https://doi.org/10.1021/ja01192a515

Surrey, A. R. (1967). 3-[(2-Oxazolidinone-3-yl)-alkyl]-4-thiazolidinones and their preparation. U.S. Patent 3309377.

Suryawanshi, R., Jadhav, S., Makwana, N., Desai, D., Chaturbhuj, D., Sonawani, A., ... Tripathy, S. (2017). Evaluation of 4-thiazolidinone derivatives as potential reverse transcriptase inhibitors against HIV-1 drug resistant strains. Bioorganic Chem., 71, 211-218. https://doi.org/10.1016/j.bioorg.2017.02.007

Tierney, J., Sheridan, D., Mascavage, L., Gorbecheva, D., Ripp, M., \& Son, S. (2005). A preliminary study on predicting ${ }^{13} \mathrm{C}$ chemical shifts for a series of disubstituted 2,3-diphenyl-1,3-thiazolidin-4-ones. Heterocyclic Communications, 11(3-4), 215-222. https://doi.org/10.1515/hc.2005.11.3-4.215

Tripathi, A. C., Gupta, S. J., Fatima, G. N., Sonar, P. K., Verma, A. \& Saraf, S. K. (2014). 4-Thiazolidinones: the advances continue.... Eur. J. Med. Chem., 72, 52-77. https://doi.org/10.1016/j.ejmech.2013.11.017

Trost, B. M., \& Curran, D. P. (1981). Chemoselective oxidation of sulfides to sulfones with potassium hydrogen persulfate. Tet. Lett., 22, 1287-1290. https://doi.org/10.1016/S0040-4039(01)90298-9

Troutman, H. D., \& Long, L. M. (1948). The synthesis of 2,3-disubstituted-4-thiazolidones. J. Am. Chem. Soc., 70, 3436-3439. https://doi.org/10.1021/ja01190a064

Webb, K. S. (1994). A mild, inexpensive and practical oxidation of sulfides. Tet. Lett., 35, 3457-3460. https://doi.org/10.1016/S0040-4039(00)73209-6

Yu, B., Liu, A. H., He, L. N., Li, B., Diao, Z. F., \& Li, Y. N. (2012). Catalyst-free approach for solvent-dependent selective oxidation of organic sulfides with oxone. Green Chem., 14, 957. https://doi.org/10.1039/c2gc00027j

\section{Copyrights}

Copyright for this article is retained by the author(s), with first publication rights granted to the journal.

This is an open-access article distributed under the terms and conditions of the Creative Commons Attribution license (http://creativecommons.org/licenses/by/4.0/). 